\title{
ВMJ Global Health Self-sampling for human papillomavirus (HPV) testing: a systematic review and meta-analysis
}

\author{
Ping Teresa Yeh, ${ }^{1}$ Caitlin E Kennedy, ${ }^{1}$ Hugo de Vuyst, ${ }^{2,3}$ Manjulaa Narasimhan ${ }^{3}$
}

To cite: Yeh PT, Kennedy CE, de Vuyst $\mathrm{H}$, et al. Self-sampling for human papillomavirus (HPV) testing: a systematic review and meta-analysis. BMJ Global Health

2019;4:e001351. doi:10.1136/ bmjgh-2018-001351

Handling editor Sanni Yaya

- Additional material is published online only. To view please visit the journal online (http://dx.doi.org/10. 1136bmjgh-2018-001351).

Received 6 December 2018 Revised 26 April 2019 Accepted 27 April 2019

Check for updates

(c) World Health Organization 2019. Licensee BMJ.

${ }^{1}$ Department of International Health, Johns Hopkins Bloomberg School of Public Health, Baltimore, Maryland, USA

${ }^{2}$ Infections and Cancer Epidemiology Group, International Agency for Research on Cancer, Lyon, France

${ }^{3}$ Department of Reproductive Health and Research, Organisation mondiale de la Santé, Geneve, Switzerland

Correspondence to Dr Manjulaa Narasimhan; narasimhanm@who.int

\section{ABSTRACT}

Introduction Human papillomavirus (HPV) self-sampling test kits may increase screening for and early detection of cervical cancer and reduce its burden globally. To inform WHO self-care guidelines, we conducted a systematic review and meta-analysis of HPV self-sampling among adult women on cervical (pre-)cancer screening uptake, screening frequency, social harms/adverse events and linkage to clinical assessment/treatment.

Methods The included studies compared women using cervical cancer screening services with HPV self-sampling with women using standard of care, measured at least one outcome, and were published in a peer-reviewed journal. We searched PubMed, the Cumulative Index to Nursing and Allied Health Literature (CNIAHL), Latin American and Caribbean Health Sciences Literature (LILACS) and Embase through October 2018. Risk of bias was assessed using the Cochrane tool for randomised controlled trials (RCTs) and the Evidence Project tool for non-randomised studies. Meta-analysis was conducted using random-effects models to generate pooled estimates of relative risk (RR). Results 33 studies in 34 articles with 369017 total participants met the inclusion criteria: 29 RCTs and 4 observational studies. All studies examined HPV selfsampling; comparison groups were standard of care (eg, Pap smear, visual inspection with acetic acid, cliniciancollected HPV testing). 93\% of participants were from high-income countries. All 33 studies measured cervical cancer screening uptake. Meta-analysis found greater screening uptake among HPV self-sampling participants compared with control (RR: $2.13,95 \% \mathrm{Cl} 1.89$ to 2.40 ). Effect size varied by HPV test kit dissemination method, whether mailed directly to home (RR: $2.27,95 \% \mathrm{Cl} 1.89$ to 2.71 ), offered door-to-door (RR: $2.37,95 \% \mathrm{Cl} 1.12$ to 5.03 ) or requested on demand (RR: $1.28,95 \% \mathrm{Cl} 0.90$ to 1.82). Meta-analysis showed no statistically significant difference in linkage to clinical assessment/treatment between arms (RR: $1.12,95 \% \mathrm{Cl} 0.80$ to 1.57). No studies measured screening frequency or social harms/adverse events.

Conclusion A growing evidence base, mainly from highincome countries and with significant heterogeneity, suggests HPV self-sampling can increase cervical cancer screening uptake compared with standard of care, with a marginal effect on linkage to clinical assessment/ treatment.

Systematic review registration number PROSPERO CRD42018114871.
Key questions

What is already known?

- Cervical cancer is one of the most common types of cancer among women globally and the leading cause of cancer deaths in women in low-income and middle-income countries, but early detection and treatment of precancerous lesions can prevent cervical cancer.

- High-risk human papillomavirus (HPV) testing is a relatively new, reasonably accurate method of secondary cervical cancer prevention.

What are the new findings?

- Meta-analysis shows that self-sampling for HPV testing may increase population uptake of cervical cancer screening, especially when HPV self-sampling kits were sent directly to women's homes or offered door-to-door by a health worker.

- However, linkage to follow-up testing and treatment after HPV self-sampling and after regular screening services alike is limited.

What do the new findings imply?

- A WHO recommendation on self-sampling for HPV may increase screening coverage (although strategies for improving linkage to treatment after positive test results are needed) and decrease the burden of cervical cancer.

\section{INTRODUCTION}

Cervical cancer is one of the most common types of cancer among women globally; in low-income and middle-income countries (LMICs), it is the leading cause of cancer deaths in women. ${ }^{12}$ Cervical cancer develops from persistent high-risk human papillomavirus (HPV) infection. ${ }^{3}$ Although vaccines exist that protect against infection and disease associated with specific types of HPV, many women in LMICs do not have access to HPV immunisation and die of this preventable cancer. ${ }^{3}$ Secondary prevention measures include early detection and treatment of precancerous lesions. Cervical cancer screening has successfully reduced cervical cancer incidence and mortality, especially 
in high-income settings with effective population-based screening programmes with good organisation, coverage and quality. ${ }^{4}$ In organised screening programmes, the majority of new cases are found in women who are never screened or underscreened; these women are often diagnosed at a later or more advanced-stage cancer, requiring more invasive treatment and leading to lower quality of life and survival. ${ }^{6}$ However, screening programmes require that a cervical cancer screening test-such as cervical cytology (Pap smear), visual inspection with acetic acid (VIA) or HPV testing-is available and accessible.

Unfortunately, in many countries, standard cervical cancer screening tests are not universally or even widely available: although almost $81 \%$ of countries have cervical cancer policies and strategies, only $48 \%$ have an operational plan with funding. ${ }^{7}$ The magnitude of this public health problem necessitates innovative approaches to support women, families and communities. Reaching more at-risk women with HPV testing, including women living with HIV who have higher risk of HPV infection and cervical cancer, is critical. ${ }^{8}$ Additionally, the two-decade to four-decade lag time between the peak of HPV infection and the peak of cervical cancer incidence leaves great opportunity for prevention. ${ }^{3}$ Primary high-risk HPV testing is a relatively new method of secondary cervical cancer prevention. $^{9-12}$ A 2018 review found that, compared with clinician samples, self-collected HPV samples showed reasonably high diagnostic accuracy, although assays based on PCR showed better performance than those based on signal amplification. ${ }^{12}$ HPV testing through self-collected specimens has gained attention for its potential to increase screening participation. Self-sampling requires individuals to obtain a kit, collect their own samples and send their specimens to a laboratory; the laboratory tests the self-collected specimens and returns the test result to the individual. ${ }^{13}$ Self-sampling can be conducted either at the clinic or outside the health system and can be initiated either by health providers or by the clients themselves. Accountability of and linkage to the health sector are important considerations for the success of this self-care intervention, including providing quality test kits, recollecting kits equitably and ensuring follow-up after self-sampling kit usage. Four systematic reviews of randomised trials in the context of population-based screening programmes showed that offering high-risk HPV self-sampling to never-screened and underscreened women increased participation compared with inviting women to have samples taken by health professionals. ${ }^{12} 14-16$

In order to develop normative guidance by the WHO on self-care interventions for sexual and reproductive health and rights, we conducted a systematic review of available evidence on the effectiveness of HPV self-sampling on increasing uptake and frequency of cervical cancer screening, social harms/adverse events, and linkage to care after a positive screening result.

\section{METHODS}

We conducted this systematic review in accordance with the Preferred Reporting Items for Systematic Reviews and Meta-Analyses guidelines. ${ }^{17} \mathrm{~A}$ full protocol is available on the PROSPERO database.

\section{Definition}

HPV self-sampling is a process where a woman who wants to know whether she has HPV infection uses a kit to collect a (cervico-) vaginal sample, which is then sent for analysis by a laboratory. Collection methods include lavage, brush, swab and vaginal patch. While HPV self-sampling cannot provide a diagnosis of cervical (pre-) cancer, it identifies those women at higher risk. The privacy afforded by self-sampling may encourage more people to get tested compared with Pap smears that, especially in LMICs, still have low coverage due to limited population awareness and lack of availability.

\section{Research question and inclusion criteria}

This review addressed the following research question: Should HPV self-sampling be offered as an additional approach to clinician-based sampling and cervical cancer screening services, including cervical screening by cytology (Pap smears) or VIA testing services?

\section{Population}

Women aged 30-60.

\section{Intervention}

Cervical screening services that include HPV self-sampling.

\section{Comparison}

Cervical screening services that do not include HPV self-sampling (eg, cervical screening by cytology, VIA testing services, clinician-collected primary HPV testing).

\section{Outcomes}

1. Uptake of cervical cancer screening services (ie, population coverage, or proportion of those offered HPV testing or other screening methods who accepted and completed screening);

2. Frequency of cervical cancer screening (ie, frequency of self-sampling compared with frequency of standard of care);

3. Social harms/adverse events (eg, device-related issues, coercion, violence (including intimate partner violence, violence from family members or community members and so on), psychosocial harm, selfharm, suicide, stigma, discrimination, frequency of testing for sexually transmitted infections and HIV) and whether these harms were corrected/had redress available; and

4. Linkage to clinical assessment or treatment of cervical lesions following a positive self-test result and positive diagnosis for HPV by a healthcare provider (ie, among people who have a positive test result, the percentage who reach this next stage of management). 
To be included in the review, an article had to (1) have a comparative study design examining women who received HPV self-sampling services with women who received HPV testing services through another modality, or to another screening test or to no intervention (ie, no cervical cancer screening services); (2) evaluate one or more of the outcomes listed above; and (3) be published in a peer-reviewed journal.

Inclusion was not restricted by location of the intervention. No language or publication date restrictions were used in the search.

\section{Search strategy and screening process}

We searched the electronic databases PubMed, the Cumulative Index to Nursing and Allied Health Literature (CINAHL), Latin American and Caribbean Health Sciences Literature (LILACS) and Embase through 19 October 2018 using search terms for HPV and self-sampling. The full search strategy is available in online supplementary file 1. Secondary reference searching was also conducted on all studies included in the review and on four relevant systematic reviews. ${ }^{12}{ }^{14-16}$ Ongoing randomised controlled trials (RCTs) were searched for through ClinicalTrials.gov, the WHO International Clinical Trials Registry Platform, the Pan African Clinical Trials Registry and the Australian New Zealand Clinical Trials Registry.

After initial title-abstract screening, full-text articles were obtained of all potential studies. Two reviewers independently assessed all full-text articles for study inclusion eligibility and resolved differences through consensus.

\section{Data extraction and analysis}

Two reviewers independently extracted data and conducted the quality assessments. Standardised data extraction forms included fields for study citation, objectives, location, population characteristics, description of the type of HPV sampling, description of any additional intervention components, study design, sample size, follow-up periods and loss to follow-up, analytic approach, reported numerical outcomes, results, and limitations.

For RCTs, risk of bias was assessed using the Cochrane Collaboration's tool for assessing risk of bias. ${ }^{18}$ For observational studies that presented comparative data, study rigour was assessed using the Evidence Project risk of bias tool for intervention evaluations. ${ }^{19}$

Where available, comparative data were stratified by the following categories: supervised (defined as direct support through inperson demonstration of how to perform self-sampling and follow-up support, counselling, referral and linkage to care) versus unsupervised (defined as indirect support through video, instructions for use, package inserts, hotline, or other information which provides counselling, support, referral and linkage to care); age ( $<50$ vs $\geq 50$ years); vulnerabilities (relating to poverty, disability, living with HIV, sex work or literacy); and high-income versus low-income or middle-income countries.
Data were analysed according to coding categories and outcomes. Where multiple studies reported the same outcome, we conducted meta-analysis using random-effects models to generate pooled relative risk (RR) using the Comprehensive Meta-Analysis program. ${ }^{20}$ Where studies reported both intention-to-treat and per-protocol analyses, we used intention-to-treat data. Where studies reported multiple follow-up periods, we used the longest follow-up time. Heterogeneity was assessed using both $\mathrm{Q}$ and I-squared statistics, and funnel plots were created to examine the potential for publication bias. Data from RCTs and non-randomised studies were analysed separately.

For the uptake of cervical cancer screening outcome, we combined authors' reported data on screening participation, attendance, response and compliance. Where studies reported uptake for conventional cytology (Pap smears), VIA or clinician-collected HPV testing in addition to HPV self-sampling, we combined uptake of any cervical cancer screening method reported among the HPV self-sampling group. Where more than one control group was reported, we compared the intervention group with the least intensive sampling strategy control group.

\section{Patient and public involvement}

A representative from a community of women living with HIV in India reviewed the manuscript for readability and accuracy and offered discussion points. Patients are currently involved in a global survey of values and preferences and focus group discussions with vulnerable communities, conducted to inform the WHO self-care guideline; they thus play a significant role in the overall recommendation outcome from this review.

\section{RESULTS}

Electronic database searching retrieved 1847 citations. Searching trial registries, contacting experts in the field and secondary searching identified an additional 11 citations (figure 1). After removing duplicates, there were 1099 unique citations. After initial screening of titles and abstracts, 361 citations remained. After two independent reviewers screened in duplicate and gained consensus, 149 articles were pulled for full-text review. Of these, 33 studies (reported in 34 articles) were included in the review. $^{21-54}$

\section{Study characteristics}

Table 1 presents summary characteristics of the 34 included articles. The 33 studies included 369017 total participants; individual study sample sizes ranged from 63 to 36 390. Articles were published between 2007 and 2018; two-thirds were published in the last 4 years. Studies were primarily conducted in high-income countries (one article each in Argentina, Australia, Belgium, Slovenia and Switzerland; two in England, Finland and Italy; three in Canada, Denmark, France and the Netherlands; four in Sweden and the USA). One study each took place in Mexico, Nigeria and Uganda. Of the included women, 


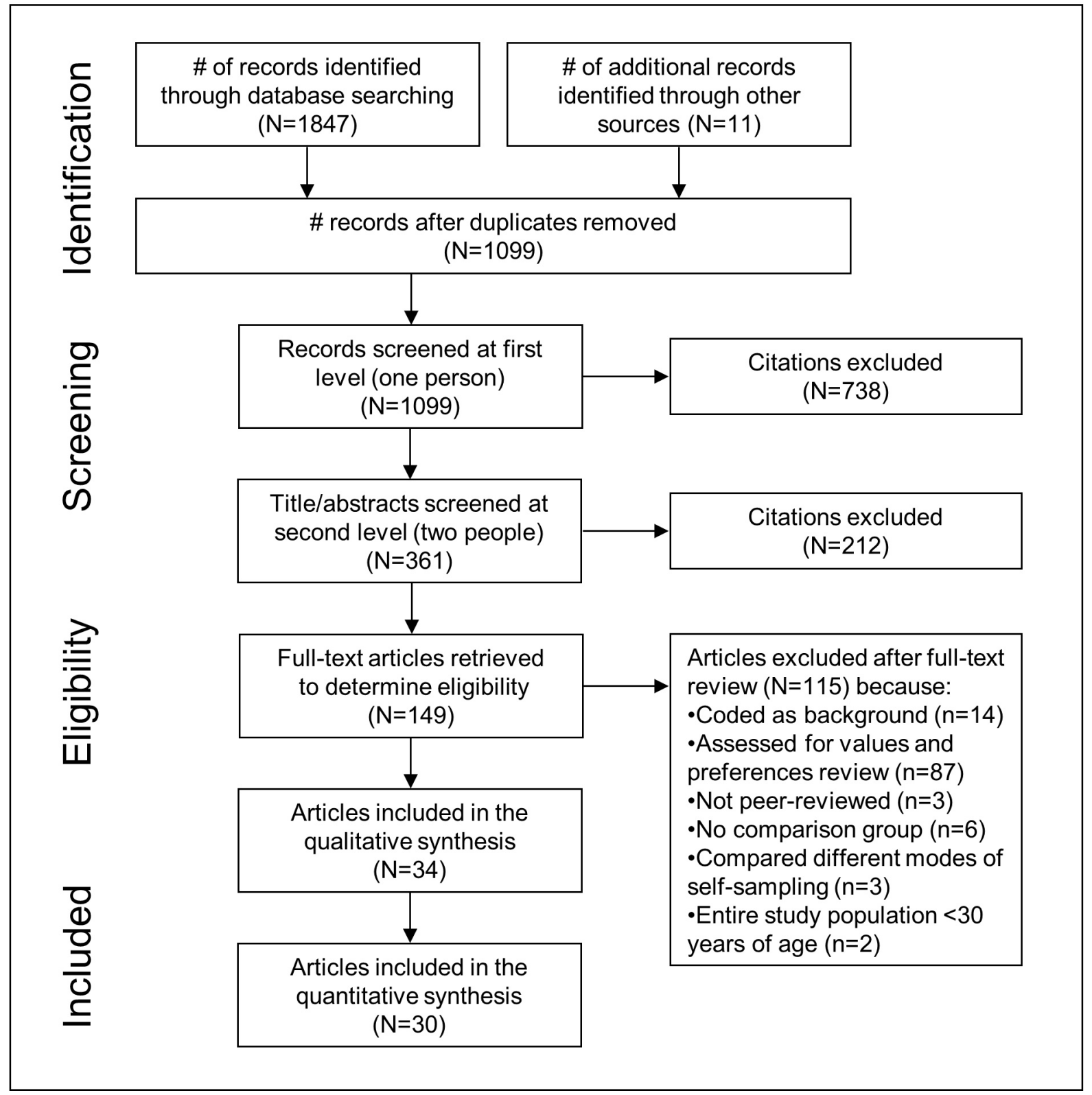

Figure 1 PRISMA flow diagram of the different phases of a systematic review. PRISMA, Preferred Reporting Items for Systematic Reviews and Meta-Analyses.

93\% were from high-income countries. Studies occurred in urban $(\mathrm{n}=14)$, mixed urban/rural $(\mathrm{n}=15)$ and rural settings $(n=5)$.

All studies included a study arm where women used cervical cancer screening services with HPV self-sampling (cervicovaginal sampling devices included 15 brush, 12 swab, 6 lavage and 3 not reported) and a control group with standard of care (eg, standard reminder letters/ recommendation to get screened, Pap smears/conventional cytology, VIA or clinician-collected HPV testing). Most studies implemented opt-out HPV self-sampling by sending an HPV self-test kit directly to participants' home addresses $(n=24)$. Six studies used opt-out HPV self-sampling offered door-to-door by a health worker (either a nurse, community health worker or research staff). Seven studies had participants opt in to HPV self-sampling by requesting a test kit by phone, mail, text message or website, or by picking up a test kit from a local pharmacy or health centre. In one study, participants self-collected samples for HPV testing in an HIV clinic.

Study participants typically comprised adult women from the general population, most often non-respondents in routine population-based cervical cancer screening programmes. Five studies were conducted among immigrant women, ethnic minorities and women from medically underserved/low socioeconomic status regions. One study each took place among women from First Nations communities and women living with HIV. Participants' ages ranged from 18 to 70 , but participants were most often between 30 and 60 years old. All studies measured uptake of cervical cancer screening, but follow-up periods ranged from immediately after being 
Table 1 Description of included studies and reported outcomes

\begin{tabular}{|c|c|c|c|c|}
\hline Author, year & $\begin{array}{l}\text { Study location and population } \\
\text { characteristics }\end{array}$ & Intervention description & Study methods & Outcomes \\
\hline Arrossi et al, $2015^{21}$ & $\begin{array}{l}\text { Argentina. } \\
\text { Urban and rural. } \\
\text { Women, general population. } \\
\text { Age: } 30+\text {. }\end{array}$ & $\begin{array}{l}\text { Intervention: self-collected HPV } \\
\text { test kit offered by CHW at home } \\
\text { visit (collected by CHW). } \\
\text { Cervical specimen, collected at } \\
\text { home, unsupervised. } \\
\text { Device: Qiagen test kit (brush). } \\
\text { Control: advice to attend health } \\
\text { clinic for cervical screening by } \\
\mathrm{CHW} \text { at home visit. }\end{array}$ & $\begin{array}{l}\text { Study design: RCT. } \\
\text { Sample size: } 6013 \\
\text { (intervention: } 3049 \text {; control: } \\
\text { 2964). } \\
\text { Length of follow-up: } 6 \\
\text { months. } \\
\text { Study name: EMA study. }\end{array}$ & $\begin{array}{l}\text { Uptake of HPV } \\
\text { testing services. }\end{array}$ \\
\hline Bais et al, $2007^{22}$ & $\begin{array}{l}\text { The Netherlands. } \\
\text { Urban. } \\
\text { Women, non-respondents to } \\
\text { regular screening programme. } \\
\text { Age: } 30-50 \text {. }\end{array}$ & $\begin{array}{l}\text { Intervention: HPV self-sampling } \\
\text { kit directly mailed to home } \\
\text { address with prepaid return } \\
\text { envelope. } \\
\text { Cervicovaginal specimen, } \\
\text { collected at home, unsupervised. } \\
\text { Device: Viba-Brush. } \\
\text { Control: reminder letter for } \\
\text { regular cytology screening. }\end{array}$ & $\begin{array}{l}\text { Study design: RCT. } \\
\text { Sample size: } 2636 \\
\text { (intervention: } 2352 \text {; control: } \\
\text { 284). } \\
\text { Length of follow-up: } 6 \\
\text { months. }\end{array}$ & $\begin{array}{l}\text { Uptake of HPV } \\
\text { testing services. }\end{array}$ \\
\hline Broberg et al, $2014^{23}$ & $\begin{array}{l}\text { Sweden. } \\
\text { Urban and rural. } \\
\text { Women, non-respondents to } \\
\text { regular screening programme. } \\
\text { Age: } 30-62 \text {. }\end{array}$ & $\begin{array}{l}\text { Intervention: HPV self-sampling } \\
\text { kit directly mailed to home } \\
\text { address with prepaid return } \\
\text { envelope. } \\
\text { Cervicovaginal specimen, } \\
\text { collected at home, unsupervised. } \\
\text { Device: QvinTip (brush). } \\
\text { Control: screening reminder } \\
\text { letter. } \\
\text { Second control: screening } \\
\text { reminder letter + telephone call } \\
\text { offering appointment for Pap } \\
\text { smear. }\end{array}$ & $\begin{array}{l}\text { Study design: RCT. } \\
\text { Sample size: } 8800 \\
\text { (intervention: } 800 \text {; control: } \\
\text { 4000; second control: } 4000 \text { ). } \\
\text { Length of follow-up: } 12 \\
\text { months. } \\
\text { Study name: RACOMIP } \\
\text { (randomized controlled } \\
\text { trial to study methods to } \\
\text { increase participation in the } \\
\text { cervical cancer screening } \\
\text { program). }\end{array}$ & $\begin{array}{l}\text { Uptake of HPV } \\
\text { testing services. }\end{array}$ \\
\hline Cadman et al, $2015^{24}$ & $\begin{array}{l}\text { England. } \\
\text { Urban and rural. } \\
\text { Women, non-respondents to } \\
\text { regular screening programme. } \\
\text { Age: } 30-64 \text {. }\end{array}$ & $\begin{array}{l}\text { Intervention: HPV self-sampling } \\
\text { kit directly mailed to home } \\
\text { address. } \\
\text { Cervicovaginal specimen, } \\
\text { collected at home, unsupervised. } \\
\text { Device: Dacron swab. } \\
\text { Control: screening reminder } \\
\text { letter (Pap test). }\end{array}$ & $\begin{array}{l}\text { Study design: RCT. } \\
\text { Sample size: } 6000 \\
\text { (intervention: } 3000 \text {; control: } \\
\text { 3000). } \\
\text { Length of follow-up: } 3 \\
\text { months. }\end{array}$ & $\begin{array}{l}\text { Uptake of HPV } \\
\text { testing services. } \\
\text { Linkage to clinical } \\
\text { assessment or } \\
\text { HPV treatment. }\end{array}$ \\
\hline $\begin{array}{l}\text { Carrasquillo et al, } \\
2018^{25}\end{array}$ & $\begin{array}{l}\text { USA. } \\
\text { Urban and rural. } \\
\text { Women, non-respondents to } \\
\text { regular screening programme, } \\
\text { ethnic minorities. } \\
\text { Age: } 30-65 \text {. }\end{array}$ & $\begin{array}{l}\text { Intervention: HPV self-sampling } \\
\text { kit and education offered door- } \\
\text { to-door by CHW (return to CHW) } \\
\text { or CHW-facilitated navigation to } \\
\text { Pap smear. } \\
\text { Cervicovaginal specimen, } \\
\text { collected at clinic, supervised. } \\
\text { Device: NR. } \\
\text { Control: CHW outreach and } \\
\text { provision of culturally tailored } \\
\text { cervical cancer screening } \\
\text { information. } \\
\text { Second control: CHW-facilitated } \\
\text { navigation to Pap smear. }\end{array}$ & $\begin{array}{l}\text { Study design: RCT. } \\
\text { Sample size: } 601 \\
\text { (intervention: } 207 \text {; control: } \\
\text { 182; second control: } 212 \text { ). } \\
\text { Length of follow-up: } 6 \\
\text { months. }\end{array}$ & $\begin{array}{l}\text { Uptake of HPV } \\
\text { testing services. }\end{array}$ \\
\hline
\end{tabular}


Table 1 Continued

\begin{tabular}{|c|c|c|c|c|}
\hline Author, year & $\begin{array}{l}\text { Study location and population } \\
\text { characteristics }\end{array}$ & Intervention description & Study methods & Outcomes \\
\hline Castle et al, $2011^{26}$ & $\begin{array}{l}\text { USA. } \\
\text { Rural. } \\
\text { Women, non-respondents to } \\
\text { regular screening programme, } \\
\text { medically underserved, ethnic } \\
\text { minorities. } \\
\text { Age: } 26-65 \text {. }\end{array}$ & $\begin{array}{l}\text { Intervention: HPV self-sampling } \\
\text { kit and education offered door- } \\
\text { to-door by study staff (return } \\
\text { immediately to staff or mail } \\
\text { later). } \\
\text { Cervicovaginal specimen, } \\
\text { collected at home, unsupervised. } \\
\text { Device: novel self-sampling kit } \\
\text { (brush). } \\
\text { Control: voucher for free } \\
\text { Pap test and staff-facilitated } \\
\text { appointment at local clinic. }\end{array}$ & $\begin{array}{l}\text { Study design: prospective } \\
\text { cohort. } \\
\text { Sample size: } 119 \\
\text { (intervention: } 77 \text {; control: } \\
\text { 42). } \\
\text { Length of follow-up: } 1 \\
\text { month. }\end{array}$ & $\begin{array}{l}\text { Uptake of HPV } \\
\text { testing services. }\end{array}$ \\
\hline
\end{tabular}

$\begin{array}{ll}\text { Darlin et al, } 2013^{27} & \text { Sweden. } \\ & \text { Urban and rural. } \\ & \text { Women, non-respondents to } \\ & \text { regular screening programme. } \\ & \text { Age: } 32-65 .\end{array}$

Intervention: HPV self-

sampling kit directly mailed to

home address with prepaid

preaddressed return envelope.

Vaginal specimen, collected at

home, unsupervised.

Device: dry cotton swab.

Control: screened by healthcare

provider (Pap test) at an

outpatient clinic (flexible, no-fee appointments). A reminder was sent to non-responders.

Duke et al, $2015^{28} \quad$ Canada.

Rural.

Women, general population. Age: 30-69.

Intervention: HPV self-sampling

kit available for pick-up at public locations (eg, hospital, pharmacies, hair salons, women's exercise centres) or research nurse dropoff at woman's house or work; return via dropoff at hospital or research nurse pick-up at participant's convenience) and Pap test and cervical cancer education/promotion campaign. Cervicovaginal specimen, collected at clinic, supervised. Device: Dacron swab. Control: Pap test and regular provincial education campaign. Second control: Pap test and cervical cancer education/ promotion campaign.

\begin{tabular}{|c|c|c|c|c|}
\hline $\begin{array}{l}\text { Giorgi Rossi et al, } \\
2011^{30}\end{array}$ & $\begin{array}{l}\text { Italy. } \\
\text { Urban and rural. } \\
\text { Women, non-respondents to } \\
\text { regular screening programme. } \\
\text { Age: } 35-65 .\end{array}$ & $\begin{array}{l}\text { Intervention: HPV self-sampling } \\
\text { kit directly mailed to home } \\
\text { address with prepaid return } \\
\text { envelope. } \\
\text { Cervicovaginal specimen, } \\
\text { collected at home, unsupervised. } \\
\text { Device: PantaRhei sampler } \\
\text { (lavage). } \\
\text { Second intervention: HPV self- } \\
\text { sampling invitation sent by mail } \\
\text { (phone order; receive kit by } \\
\text { mail or pick up at clinic, prepaid } \\
\text { return envelope). } \\
\text { Control: screening reminder } \\
\text { letter (Pap test). } \\
\text { Second control: screening } \\
\text { reminder letter (HPV test). }\end{array}$ & $\begin{array}{l}\text { Study design: RCT. } \\
\text { Sample size: } 2480 \\
\text { (intervention: } 616 \text {; second } \\
\text { intervention: } 622 \text {; control: } \\
\text { 619; second control: } 616 \text { ). } \\
\text { Length of follow-up: } 5 \\
\text { months. }\end{array}$ & $\begin{array}{l}\text { Uptake of HPV } \\
\text { testing services. }\end{array}$ \\
\hline
\end{tabular}

Study design: RCT. Uptake of HPV Sample size: $1500 \quad$ testing services. (intervention: 1000; control: 500).

Length of follow-up: NR.

Study design: prospective cohort.

Sample size: 6285 (baseline:

Uptake of HPV intervention: 1928; control: 1524 second control: 2833; endline: intervention: 1760; control: 1536; second control: 2761).

Length of follow-up: 24 months. 
Table 1 Continued

\begin{tabular}{lllll}
\hline Author, year & $\begin{array}{l}\text { Study location and population } \\
\text { characteristics }\end{array}$ & Intervention description & Study methods & Outcomes \\
\hline Giorgi Rossi et al, & Italy. & Intervention: HPV self-sampling & Study design: RCT. & \\
$2015^{29}$ & Urban and rural. & kit directly mailed to home & Sample size: 14 041 & Uptake of HPV \\
& Women, non-respondents to & address, preceded by a & (intervention: 4516 ; second & testing services. \\
& regular screening programme. & notification. & intervention: $4513 ;$ control: Linkage to clinical \\
& Age: 30-64. & Cervicovaginal specimen, & 5012). & assessment or \\
& & collected at home, unsupervised. Length of follow-up: 3 & HPV treatment.
\end{tabular}

Second intervention: HPV self-

sampling kit available for pick-up

at local pharmacies.

Control: screening reminder

letter (Pap test or HPV test).

\begin{tabular}{|c|c|c|c|c|}
\hline Gok et al, $2010^{31}$ & $\begin{array}{l}\text { The Netherlands. } \\
\text { Urban. } \\
\text { Women, non-respondents to } \\
\text { regular screening programme. } \\
\text { Age: } 30-60 \text {. }\end{array}$ & $\begin{array}{l}\text { Intervention: HPV self-sampling } \\
\text { kit directly mailed to home } \\
\text { address, preceded by a } \\
\text { notification. } \\
\text { Cervicovaginal specimen, } \\
\text { collected at home, unsupervised. } \\
\text { Device: Delphi Screener (lavage). } \\
\text { Control: screening reminder } \\
\text { letter (Pap test). }\end{array}$ & $\begin{array}{l}\text { Study design: RCT. } \\
\text { Sample size: } 28073 \\
\text { (intervention: } 27792 \text {; } \\
\text { control: } 281 \text { ). } \\
\text { Length of follow-up: } 12 \\
\text { months. } \\
\text { Study name: PROHTECT-1 } \\
\text { (PRotection by Offering HPV } \\
\text { TEsting on Cervicovaginal } \\
\text { specimens Trial). }\end{array}$ & $\begin{array}{l}\text { Uptake of HPV } \\
\text { testing services. }\end{array}$ \\
\hline Gök et al, $2012^{32}$ & $\begin{array}{l}\text { The Netherlands. } \\
\text { Urban. } \\
\text { Women, non-respondents to } \\
\text { regular screening programme. } \\
\text { Age: } 30-60 \text {. }\end{array}$ & $\begin{array}{l}\text { Intervention: HPV self-sampling } \\
\text { kit directly mailed to home } \\
\text { address. } \\
\text { Vaginal specimen, collected at } \\
\text { home, unsupervised. } \\
\text { Device: Viba-Brush. } \\
\text { Control: screening reminder } \\
\text { letter (Pap test). }\end{array}$ & $\begin{array}{l}\text { Study design: RCT. } \\
\text { Sample size: } 26409 \\
\text { (intervention: } 26145 \text {; } \\
\text { control: 264). } \\
\text { Length of follow-up: } 12 \\
\text { months. } \\
\text { Study name: PROHTECT-2 } \\
\text { (PRotection by Offering HPV } \\
\text { TEsting on Cervicovaginal } \\
\text { specimens Trial). }\end{array}$ & $\begin{array}{l}\text { Uptake of HPV } \\
\text { testing services. }\end{array}$ \\
\hline $\begin{array}{l}\text { Gustavsson et al, } \\
2018^{33}\end{array}$ & $\begin{array}{l}\text { Sweden. } \\
\text { Urban and rural. } \\
\text { Women, non-respondents to } \\
\text { regular screening programme. } \\
\text { Age: } 30-49 .\end{array}$ & $\begin{array}{l}\text { Intervention: HPV self-sampling } \\
\text { kit directly mailed to home } \\
\text { address with preaddressed } \\
\text { return envelope. } \\
\text { Vaginal specimen, collected at } \\
\text { home, unsupervised. } \\
\text { Device: Viba-Brush. } \\
\text { Control: screened by healthcare } \\
\text { provider (Pap test). }\end{array}$ & $\begin{array}{l}\text { Study design: RCT. } \\
\text { Sample size: } 36390 \\
\text { (intervention: } 17997 ; \\
\text { control: } 18 \text { 393). } \\
\text { Length of follow-up: } 18 \\
\text { months. }\end{array}$ & $\begin{array}{l}\text { Uptake of HPV } \\
\text { testing services. } \\
\text { Linkage to clinical } \\
\text { assessment or } \\
\text { HPV treatment. }\end{array}$ \\
\hline $\begin{array}{l}\text { Haguenoer et al, } \\
2014^{34}\end{array}$ & $\begin{array}{l}\text { France. } \\
\text { Urban and rural. } \\
\text { Women, non-respondents to } \\
\text { regular screening programme. } \\
\text { Age: } 30-65 .\end{array}$ & $\begin{array}{l}\text { Intervention: HPV self-sampling } \\
\text { kit directly mailed to home } \\
\text { address. } \\
\text { Vaginal specimen, collected at } \\
\text { home, unsupervised. } \\
\text { Device: dry nylon flocked swab. } \\
\text { Control: screening reminder } \\
\text { letter (Pap test) and phone call } \\
\text { reminder. } \\
\text { Second control: no intervention. }\end{array}$ & $\begin{array}{l}\text { Study design: RCT. } \\
\text { Sample size: } 5998 \\
\text { (intervention: 1999; control: } \\
\text { 2000; second control: 1999). } \\
\text { Length of follow-up: } 12 \\
\text { months. }\end{array}$ & $\begin{array}{l}\text { Uptake of HPV } \\
\text { testing services. }\end{array}$ \\
\hline Ivanus et al, $2018^{35}$ & $\begin{array}{l}\text { Slovenia. } \\
\text { Urban and rural. } \\
\text { Women, non-respondents to } \\
\text { regular screening programme. } \\
\text { Age: } 30-64 \text {. }\end{array}$ & $\begin{array}{l}\text { Intervention: HPV self-sampling } \\
\text { kit directly mailed to home } \\
\text { address. } \\
\text { Cervicovaginal specimen, } \\
\text { collected at home, unsupervised. } \\
\text { Device: QvinTip (brush), } \\
\text { HerSwab, Delphi Screening } \\
\text { (lavage). } \\
\text { Second intervention: HPV self- } \\
\text { sampling kit available by order or } \\
\text { for pick-up at local pharmacies. } \\
\text { Control: screening reminder } \\
\text { letter. }\end{array}$ & $\begin{array}{l}\text { Study design: RCT. } \\
\text { Sample size: } 26556 \\
\text { (intervention: } 9956 \text {; second } \\
\text { intervention: } 14400 \text {; control: } \\
\text { 2600). } \\
\text { Length of follow-up: } 12 \\
\text { months. }\end{array}$ & $\begin{array}{l}\text { Uptake of HPV } \\
\text { testing services. } \\
\text { Linkage to clinical } \\
\text { assessment or } \\
\text { HPV treatment. }\end{array}$ \\
\hline
\end{tabular}


Table 1 Continued

\begin{tabular}{ll}
\hline Author, year & $\begin{array}{l}\text { Study location and population } \\
\text { characteristics }\end{array}$
\end{tabular}

\begin{tabular}{lllll} 
Author, year & characteristics & Intervention description & Study methods & Outcomes \\
\hline \multirow{2}{*}{ Kellen et al, 2018 } & Belgium. & Intervention: HPV self-sampling & Study design: RCT. & Uptake of HPV \\
& Urban and rural. & kit directly mailed to home & Sample size: 35 354 & testing services. \\
& Women, non-respondents to & address with prepaid return & (intervention: 9118; second
\end{tabular}

Women, non-respondents to regular screening programme. Age: $30-64$.

$\begin{array}{ll}\text { Lam et al, } 2017^{37} & \text { Denmark. } \\ & \text { Urban. } \\ & \text { Women, non-respondents to } \\ & \text { regular screening programme. } \\ & \text { Age: } 27-65 .\end{array}$

Lazcano-Ponce et al, $2011^{38}$
Mexico.

Rural.

Women, medically underserved/ low socioeconomic status. Age: 25-65. address with prepaid return envelope.

Cervicovaginal specimen,

collected at home, unsupervised. Length of follow-up: 12

Device: Qvintip (brush).

Second intervention: HPV self-

sampling invitation sent by mail;

order kit by mail, phone, email or website.

Control: screening reminder letter (Pap test by general practitioner or gynaecologist).

Second control: no intervention.

Intervention: HPV self-sampling invitation sent by mail; order kit by mail, phone, email or website; prepaid return envelope.

Cervicovaginal specimen, collected at home, unsupervised. Device: Evalyn Brush.

Control: screened by healthcare provider (Pap test).

Intervention: HPV self-sampling kit and education offered doorto-door by nurse.

Vaginal specimen, collected at home, supervised.

Device: Digene (brush).

Control: screened by healthcare provider (Pap test).

Intervention: HPV self-sampling sectional.

Length of follow-up: 7 months.

Study name: Copenhagen Self-Sampling Initiative.

Study design: RCT.

Sample size: 25061 (intervention: 12 330; control: 12 731).

Length of follow-up: NR.

Study name: MARCH

(Mexican Appraisal of

Routine Cytology versus

vaginal HPV screening).

$\begin{aligned} \text { Modibbo et al, } 2017^{39} & \text { Nigeria. } \\ & \text { Urban. } \\ & \text { Women, general population. } \\ & \text { Age: } 30-65 .\end{aligned}$

Moses et al, $2015^{40} \quad$ Uganda.

Urban.

Women, general population. Age: $30-65$. kit directly mailed to home address with prepaid return envelope (or could drop off completed kit at designated collection points in community or at the hospital).

Cervicovaginal specimen, collected at home, unsupervised.

Device: NR.

Control: HPV testing appointment at hospital clinic.

Intervention: HPV self-sampling kit and education offered doorto-door by outreach worker (return to worker).

Cervicovaginal specimen, collected at home, unsupervised. Device: Dacron swab.

Control: Screened by healthcare provider (VIA).

\begin{tabular}{|c|c|c|c|c|}
\hline Murphy et al, $2016^{41}$ & $\begin{array}{l}\text { USA. } \\
\text { Urban. } \\
\text { Women, living with HIV. } \\
\text { Age: } 18+\text {. }\end{array}$ & $\begin{array}{l}\text { Intervention: HPV self-sampling } \\
\text { kit offered in HIV clinic. } \\
\text { Cervicovaginal specimen, } \\
\text { collected at clinic, unsupervised. } \\
\text { Device: Qiagen test kit (brush). } \\
\text { Control: screening reminder } \\
\text { letter. }\end{array}$ & $\begin{array}{l}\text { Study design: RCT. } \\
\text { Sample size: } 94 \\
\text { (intervention: } 63 \text {; control: } \\
\text { 31). } \\
\text { Length of follow-up: } 6 \\
\text { months. }\end{array}$ & $\begin{array}{l}\text { Uptake of HPV } \\
\text { testing services. }\end{array}$ \\
\hline
\end{tabular}

Study design: RCT.

Sample size: 400

Uptake of HPV (intervention: 200; control: 200).

Length of follow-up: 1 month.

testing services.
Study design: RCT.

Sample size: 500

(intervention: 250; control:

250).

Length of follow-up: NR.

Uptake of HPV testing services. Linkage to clinical assessment or HPV treatment.

Study design: cross- Uptake of HPV testing services.

Uptake of HPV

Continued 
Table 1 Continued

\begin{tabular}{llll}
\hline Author, year & $\begin{array}{l}\text { Study location and population } \\
\text { characteristics }\end{array}$ & Intervention description & Stud \\
\hline Piana et al, 201142 & France. & Intervention: HPV self- & Stud \\
& Urban. & sampling kit directly mailed to & Sam \\
& Women, non-respondents to & home address with prepaid & (in \\
& regular screening programme. & preaddressed return envelope, & 4934 \\
& Age: 35-69. & preceded by a notification with & Le \\
& & an opt-out option. & Cervicovaginal specimen, \\
& & collected at home, unsupervised. \\
& & Device: NR. \\
& Control: screening reminder \\
& & letter (Pap test).
\end{tabular}

\begin{tabular}{|c|c|c|c|c|}
\hline Racey et al, $2016^{54}$ & $\begin{array}{l}\text { Canada. } \\
\text { Rural. } \\
\text { Women, non-respondents to } \\
\text { regular screening programme. } \\
\text { Age: } 30-70 .\end{array}$ & $\begin{array}{l}\text { Intervention: HPV self-sampling } \\
\text { kit directly mailed to home } \\
\text { address with preaddressed } \\
\text { return envelope, preceded by } \\
\text { a notification with an opt-out } \\
\text { option. } \\
\text { Vaginal specimen, collected at } \\
\text { home, unsupervised. } \\
\text { Device: Dacron swab. } \\
\text { Control: screening reminder } \\
\text { letter (Pap test). } \\
\text { Second control: opportunistic } \\
\text { standard-of-care Pap test (no } \\
\text { screening reminder). }\end{array}$ & $\begin{array}{l}\text { Study design: RCT. } \\
\text { Sample size: } 818 \\
\text { (intervention: } 335 \text {; control: } \\
\text { 331; second control: 152). } \\
\text { Length of follow-up: NR. }\end{array}$ & $\begin{array}{l}\text { Uptake of HPV } \\
\text { testing services. }\end{array}$ \\
\hline $\begin{array}{l}\text { Sancho-Garnier et al, } \\
2013^{43}\end{array}$ & $\begin{array}{l}\text { France. } \\
\text { Urban. } \\
\text { Women, non-respondents to } \\
\text { regular screening programme, } \\
\text { low socioeconomic status. } \\
\text { Age: } 35-69 \text {. }\end{array}$ & $\begin{array}{l}\text { Intervention: HPV self-sampling } \\
\text { kit directly mailed to home } \\
\text { address, preceded by a } \\
\text { notification. } \\
\text { Vaginal specimen, collected at } \\
\text { home, unsupervised. } \\
\text { Device: Dacron swab. } \\
\text { Control: screened by healthcare } \\
\text { provider (Pap smear) at } \\
\text { outpatient clinic. }\end{array}$ & $\begin{array}{l}\text { Study design: RCT. } \\
\text { Sample size: } 18730 \\
\text { (intervention: } 8829 \text {; control: } \\
\text { 9901). } \\
\text { Length of follow-up: NR. }\end{array}$ & $\begin{array}{l}\text { Uptake of HPV } \\
\text { testing services. } \\
\text { Linkage to clinical } \\
\text { assessment or } \\
\text { HPV treatment. }\end{array}$ \\
\hline Sewali et al, $2015^{44}$ & $\begin{array}{l}\text { USA. } \\
\text { Urban. } \\
\text { Women, Somali immigrant. } \\
\text { Age: } 25-70 .\end{array}$ & $\begin{array}{l}\text { Intervention: HPV self-sampling } \\
\text { kit and education offered door- } \\
\text { to-door by outreach worker } \\
\text { (return to worker). } \\
\text { Cervicovaginal specimen, } \\
\text { collected at home, unsupervised. } \\
\text { Device: Just For Me (brush). } \\
\text { Control: screened by healthcare } \\
\text { provider (Pap test). }\end{array}$ & $\begin{array}{l}\text { Study design: RCT. } \\
\text { Sample size: } 63 \\
\text { (intervention: } 32 \text {; control: } \\
\text { 31). } \\
\text { Length of follow-up: } 3 \\
\text { months. }\end{array}$ & $\begin{array}{l}\text { Uptake of HPV } \\
\text { testing services. }\end{array}$ \\
\hline Szarewski et al, $2011^{46}$ & $\begin{array}{l}\text { England. } \\
\text { Urban. } \\
\text { Women, non-respondents to } \\
\text { regular screening programme. } \\
\text { Age: } 29-65 .\end{array}$ & $\begin{array}{l}\text { Intervention: HPV self- } \\
\text { sampling kit directly mailed to } \\
\text { home address with prepaid } \\
\text { preaddressed return envelope. } \\
\text { Cervicovaginal specimen, } \\
\text { collected at home, unsupervised. } \\
\text { Device: Qiagen test kit (cotton } \\
\text { swab). } \\
\text { Control: screened by healthcare } \\
\text { provider (Pap test). }\end{array}$ & $\begin{array}{l}\text { Study design: RCT. } \\
\text { Sample size: } 3000 \\
\text { (intervention: } 1500 \text {; control: } \\
\text { 1500). } \\
\text { Length of follow-up: } 6 \\
\text { months. }\end{array}$ & $\begin{array}{l}\text { Uptake of HPV } \\
\text { testing services. }\end{array}$ \\
\hline
\end{tabular}

Continued 
Table 1 Continued

\begin{tabular}{ll}
\hline Author, year & $\begin{array}{l}\text { Study location and population } \\
\text { characteristics }\end{array}$
\end{tabular}

\begin{tabular}{ll} 
Author, year & characteristics \\
\hline Tranberg et al, 2018 & Denmark. \\
& Urban and rural. \\
& Women, non-respondents to \\
& regular screening programme. \\
& Age: $30-64$.
\end{tabular}

\section{Intervention description}

Intervention: HPV self-sampling kit directly mailed to home address.

Cervicovaginal specimen, collected at home, unsupervised. 3262).

Device: Evalyn Brush. Second intervention: HPV self- months.

sampling invitation sent by mail; Study name: CHOiCE order kit by email, text message, (Cervical Home-based phone or website.

Control: screening reminder letter (Pap test).

$\begin{array}{ll}\text { Tranberg et al, } 2018^{48} & \text { Denmark. } \\ & \text { Urban and rural. } \\ & \text { Women, non-respondents to } \\ & \text { regular screening programme. } \\ & \text { Age: } 30-64 .\end{array}$

Virtanen et al, $2011^{50} \quad$ Finland

Urban.

Women, non-respondents to regular screening programme. Age: 30-60.

\section{Intervention: HPV self-sampling}

kit directly mailed to home address.

Cervicovaginal specimen, collected at home, unsupe

Device: Evalyn Brush.

Second intervention: HPV self-

order kit by email, text message, CHOiCE above.

phone or website.

Control: screening reminder letter (Pap test).

Intervention: HPV self-sampling

kit directly mailed to home address.

Cervicovaginal specimen, sampling invitation sent by mail; Study name: subanalysis of

collected at home, unsupervised. Length of follow-up: NR.

Device: Delphi Screener (lavage)

Control: screening reminder letter.

\begin{tabular}{|c|c|c|c|c|}
\hline Virtanen et al, $2015^{49}$ & $\begin{array}{l}\text { Finland. } \\
\text { Urban and rural. } \\
\text { Women, non-respondents to } \\
\text { regular screening programme. } \\
\text { Age: } 30-60 .\end{array}$ & $\begin{array}{l}\text { Intervention: HPV self-sampling } \\
\text { kit directly mailed to home } \\
\text { address. } \\
\text { Cervicovaginal specimen, } \\
\text { collected at home, unsupervised. } \\
\text { Device: Delphi Screener (lavage). } \\
\text { Control: screening reminder } \\
\text { letter. } \\
\text { Second control: screening } \\
\text { reminder letter + second } \\
\text { reminder letter. }\end{array}$ & $\begin{array}{l}\text { Study design: prospective } \\
\text { cohort. } \\
\text { Sample size: } 30827 \\
\text { (baseline/control: } 30 \\
\text { 827; timepoint 2/second } \\
\text { control: } 7397 ; \text { timepoint 3/ } \\
\text { intervention: } 4536 \text { ). } \\
\text { Length of follow-up: } 24 \\
\text { months. }\end{array}$ & $\begin{array}{l}\text { Uptake of HPV } \\
\text { testing services. }\end{array}$ \\
\hline Viviano et al, $2017^{51}$ & $\begin{array}{l}\text { Switzerland. } \\
\text { Urban. } \\
\text { Women, non-respondents to } \\
\text { regular screening programme. } \\
\text { Age: } 25-69 \text {. }\end{array}$ & $\begin{array}{l}\text { Intervention: HPV self-sampling } \\
\text { kit directly mailed to home } \\
\text { address with prepaid return } \\
\text { envelope. } \\
\text { Cervicovaginal specimen, } \\
\text { collected at home, unsupervised. } \\
\text { Device: ESwab. } \\
\text { Control: screened by healthcare } \\
\text { provider (liquid-based cytology). }\end{array}$ & $\begin{array}{l}\text { Study design: RCT. } \\
\text { Sample size: } 667 \\
\text { (intervention: } 336 \text {; control: } \\
\text { 331). } \\
\text { Length of follow-up: NR. }\end{array}$ & $\begin{array}{l}\text { Uptake of HPV } \\
\text { testing services. } \\
\text { Linkage to clinical } \\
\text { assessment or } \\
\text { HPV treatment. }\end{array}$ \\
\hline
\end{tabular}

\section{Study design: RCT.}

Sample size: 25597 (intervention: 7086; control: 18 511).

Sample size: 979 (intervention: 3265; second intervention: 3264; control:

3262)

Length of follow-up: 6

Sample size: 9791

Uptake of HPV testing services.

Uptake of HPV testing services.

Uptake of HPV testing services.

Continued 
Table 1 Continued

\begin{tabular}{|c|c|c|c|c|}
\hline Author, year & $\begin{array}{l}\text { Study location and population } \\
\text { characteristics }\end{array}$ & Intervention description & Study methods & Outcomes \\
\hline Zehbe et al, $2016^{53}$ & $\begin{array}{l}\text { Canada. } \\
\text { Rural. } \\
\text { Women, First Nations } \\
\text { communities. } \\
\text { Age: } 25-69 .\end{array}$ & $\begin{array}{l}\text { Intervention: HPV self-sampling } \\
\text { kit directly mailed to home } \\
\text { address. } \\
\text { Cervicovaginal specimen, } \\
\text { collected at home, unsupervised. } \\
\text { Device: NR. } \\
\text { Control: screened by healthcare } \\
\text { provider (Pap test). }\end{array}$ & $\begin{array}{l}\text { Study design: RCT. } \\
\text { Sample size: } 834 \\
\text { (intervention: } 404 \text {; control: } \\
430 \text { ). } \\
\text { Length of follow-up: } 3 \\
\text { months } \\
\text { Study name: ACCSS } \\
\text { (Anishinaabek Cervical } \\
\text { Cancer Screening Study). }\end{array}$ & $\begin{array}{l}\text { Uptake of HPV } \\
\text { testing services. }\end{array}$ \\
\hline
\end{tabular}

$\mathrm{CHW}$, community health worker; HPV, human papillomavirus; NR, not reported; RCT, randomised controlled trial; VIA, visual inspection with acetic acid.

offered screening to 36 months, with half between 6 and 12 months.

Study designs included 29 RCTs and 4 observational studies (3 prospective cohort studies and 1 cross-sectional study). Risk of bias in RCTs was generally low. Although it was impossible to blind participants and personnel to HPV self-sampling versus standard of care, we judged most outcomes were not likely to be influenced by lack of blinding. The observational studies were of moderate quality. Table 2 (for RCTs) and table 3 (for non-RCTs) present an assessment of study rigour. The Grading of Recommendations Assessment, Development and Evaluation (GRADE) table for this review with quality assessment by outcome is available in online supplementary file 2.

For each of the main outcomes, the results are presented below and summarised in table 4 .

\section{Uptake of cervical cancer screening services}

All 34 included articles reported some measure of uptake of cervical cancer screening. Substantial heterogeneity was present in all meta-analyses of screening uptake. Combining all self-sampling kit dissemination methods, meta-analysis of the 29 reported effect sizes from $\mathrm{RCTs}^{21-25}$ 27 29-36 38-4750-54 found that women were twice as likely to use cervical cancer screening services through self-sampling compared with standard-of-care screening practices (RR: 2.13, 95\% CI 1.89 to 2.40, I-squared: 99.34) (figure 2).

Uptake stratified by HPV self-sampling kit dissemination strategy In the 23 RCTs comparing opt-out HPV self-sampling (kit directly mailed to home address) with control, ${ }^{22-24} 27$ 29-36 $39424345-4751-54$ meta-analysis found that HPV self-sampling was associated with twice the likelihood of attending screening compared with control (RR: 2.27, 95\% CI 1.89 to 2.71, I-squared: 99.27) (figure 3). These RCTs all took place in Europe, except for two in Canada and one in Nigeria. RCT participants were all non-respondents to regular screening, except for one RCT among general population women and one among First Nations communities. All intervention arm participants self-sampled outside the clinic (ie, at home or work) without supervision using a variety of sampling kit types. One observational study showed a similar effect. In Finland, a prospective cohort study followed 31053 women identified for regular cervical cancer screening. ${ }^{49}$ A second reminder letter after initial invitation increased screening participation from $72.6 \%$ to $79.2 \%$ (95\% CI 78.8 to 79.7$)$, and sending a self-sampling test kit directly to women's home further increased overall uptake to $82.2 \%$ (95\% CI 81.8 to 82.7 ).

Six studies compared opt-out HPV self-sampling offered door-to-door by health workers with control. 212526384044 In these studies, a community health worker, nurse or health outreach worker would visit participants' homes, provide education on cervical cancer and screening, offer a self-sampling HPV test kit (brush or swab) to the participant, and collect the kit for lab testing. Meta-analysis of five RCTs ${ }^{21} 25384044$ showed that women were almost three times as likely to participate in cervical cancer screening (RR: 2.37, 95\% CI 1.12 to 5.03, I-squared: 99.72) (figure 4). Two of these RCTs took place in the USA among ethnic minority or immigrant women, one each took place in Argentina and Uganda among women in the general population, and one took place among medically underserved women from a poverty reduction programme in Mexico. One prospective cohort study on community outreach in the rural Mississippi Delta, USA, found that medically underserved non-respondents to the regular screening programme responded more to an offer of the HPV self-sampling kit and cervical cancer education $(62 / 77,80.5 \%)$ than to a voucher for a free Pap test with staff-facilitated appointment scheduling at the local clinic $(17 / 42,40.5 \%) .{ }^{26}$

Meta-analysis of five RCTs ${ }^{29} 30353647$ found a non-significant increase in likelihood of HPV screening participation for opt-in HPV self-sampling where participants requested or picked up self-sampling kits compared with control (RR: 1.28, 95\% CI 0.90 to 1.82, I-squared: 98.36) (figure 5). These RCTs all took place in Europe among women aged 30-65 who were hard to reach through conventional cervical cancer screening programmes. Women could order HPV self-sampling kits by email, text message, phone, website or mail; kits could be delivered to participants' homes or picked up at a local pharmacy or health centre. All participants in the intervention arm 
Table 2 Quality assessment of included studies (RCTs)

Cochrane risk of bias tool (for RCTs)

\begin{tabular}{|c|c|c|c|c|c|c|}
\hline Type of bias & Selection & & Performance & Detection & Attrition & Reporting \\
\hline Author, year & $\begin{array}{l}\text { Random } \\
\text { sequence } \\
\text { generation }\end{array}$ & $\begin{array}{l}\text { Allocation } \\
\text { concealment }\end{array}$ & $\begin{array}{l}\text { Blinding of } \\
\text { participants and } \\
\text { personnel }^{\star}\end{array}$ & $\begin{array}{l}\text { Blinding of } \\
\text { outcome } \\
\text { assessment* }\end{array}$ & $\begin{array}{l}\text { Incomplete } \\
\text { outcome data }\end{array}$ & Selective reporting \\
\hline Arrossi et al, $2015^{21}$ & Medium† & Low & Low & Low & Low & Low \\
\hline Bais et al, $2007^{22}$ & Low & Medium $\ddagger$ & Low & Low & Low & Low \\
\hline Broberg et al, $2014^{23}$ & Medium $\ddagger$ & Medium $\ddagger$ & Low & Low & Low & Low \\
\hline Cadman et al, $2015^{24}$ & Low & Low & Low & Low & Low & Low \\
\hline Carrasquillo et al, $2018^{25}$ & Low & Low & Low & Low & Low & Low \\
\hline Darlin et al, $2013^{27}$ & Medium $\ddagger$ & Medium $\ddagger$ & Low & Low & Low & Medium†† \\
\hline Giorgi Rossi et al, $2011^{30}$ & Low & Low & Low & Low & Low & Low \\
\hline Giorgi Rossi et al, $2015^{29}$ & Low & Low & Low & Low & Low & Low \\
\hline Gok et al, $2010^{31}$ & Low & Medium & Low & Low & Low & Low \\
\hline Gök et al, $2012^{32}$ & Low & Medium $\ddagger$ & Low & Low & Low & Medium†† \\
\hline Gustavsson et al, $2018^{33}$ & Low & Low & Low & Low & Low & Low \\
\hline Haguenoer et al, $2014^{34}$ & Low & Low & Low & Low & Low & Low \\
\hline Ivanus et al, $2018^{35}$ & Low & Low & Low & Low & Low & Low \\
\hline Kellen et al, $2018^{36}$ & Low & High§ & Low & Low & Low & Low \\
\hline Lazcano-Ponce et al, $2011^{38}$ & Medium & Low & Low & Low & Low & Medium†† \\
\hline Modibbo et al, $2017^{39}$ & $\mathrm{High}^{\star *}$ & Medium & Low & Low & Low & Medium†† \\
\hline Moses et al, $2015^{40}$ & Low & Low & Low & Low & Medium & Low \\
\hline Murphy et al, $2016^{41}$ & Low & Low & Low & Low & Low & Low \\
\hline Piana et al, $2011^{42}$ & Low & Medium $\ddagger$ & Low & Low & Medium & High†十㧊 \\
\hline Racey et al, $2016^{54}$ & Low & Low & Low & Low & Medium & Low \\
\hline Sancho-Garnier et al, $2013^{43}$ & Medium $\neq$ & Medium $\neq$ & Low & Low & Low & Medium†† \\
\hline Sewali et al, $2015^{44}$ & Low & Low & Low & Low & Low & Low \\
\hline Sultana et al, $2016^{45}$ & Low & Low & Low & Low & Low & Medium \\
\hline Szarewski et al, $2011^{46}$ & Medium $^{3}$ & Medium $^{3}$ & Low & Low & Low & Low \\
\hline Tranberg et al, $2018^{47}$ & Low & Low & Low & Low & Low & Low \\
\hline $\begin{array}{l}\text { Tranberg et al, } 2018 \\
\text { (subanalysis) }^{48}\end{array}$ & Low & Low & Low & Low & Low & Low \\
\hline Virtanen et al, $2011^{50}$ & Low & Medium $\ddagger$ & Low & Low & Low & Low \\
\hline Viviano et al, $2017^{51}$ & Low & Low & Low & Low & Low & Low \\
\hline Wikström et al, $2011^{52}$ & Medium $\ddagger$ & Medium $\ddagger$ & Low & Low & Low & Low \\
\hline Zehbe et al, $2016^{53}$ & Medium $\dagger$ & Medium & Low & Low & Medium & Medium \\
\hline
\end{tabular}

Green: low risk of bias; yellow: medium risk of bias; red: high risk of bias.

*Given the intervention of interest (self-sampling for HPV testing), blinding was not possible for participants and personnel, nor was it possible to blind for outcome assessment. However, the measured outcomes (uptake of cervical cancer screening, frequency of HPV testing, social harms/adverse events and linkage to care) are unlikely to be influenced by lack of blinding. For uptake, the outcome was measured by lab/medical records (number of kits sent in for testing and number of patients who got the Pap smear or VIA), not by self-report. For linkage to care, the outcome was measured through medical records, not by self-report.

†Cluster-randomised community health workers or communities to self-sampling and control arms.

\pm Details of the randomisation and/or allocation process are not documented.

§Timing of screening invitation was different for the intervention group compared with the control group.

INon-random factor is included in design (eg, women randomised to the self-sampling arm who were not at home when visited by researcher were reassigned to control/conventional cytology).

${ }^{* *}$ Randomisation occurred after enrolment.

††Intention to treat was not reported.

$\ddagger \ddagger$ Women in the self-sampling arm could opt out; those who did were excluded from the analysis (possibly leading to an artificially high participation rate in the self-sampling arm).

HPV, human papillomavirus; RCT, randomised controlled trial; VIA, visual inspection with acetic acid

self-sampled at home without supervision. Two observational studies presented similar increases in uptake. In Denmark, the Copenhagen Self-Sampling Initiative invited women to opt in to order a HPV self-sampling kit by mail, phone, email or website. ${ }^{37}$ Of 23632 women invited, 7484 women ordered a self-sampling brush and 4824 returned the brush for DNA testing; 2288 received screening by a physician. A 2-year prospective cohort study in Canada compared preintervention and postintervention screening rates in three communities: 


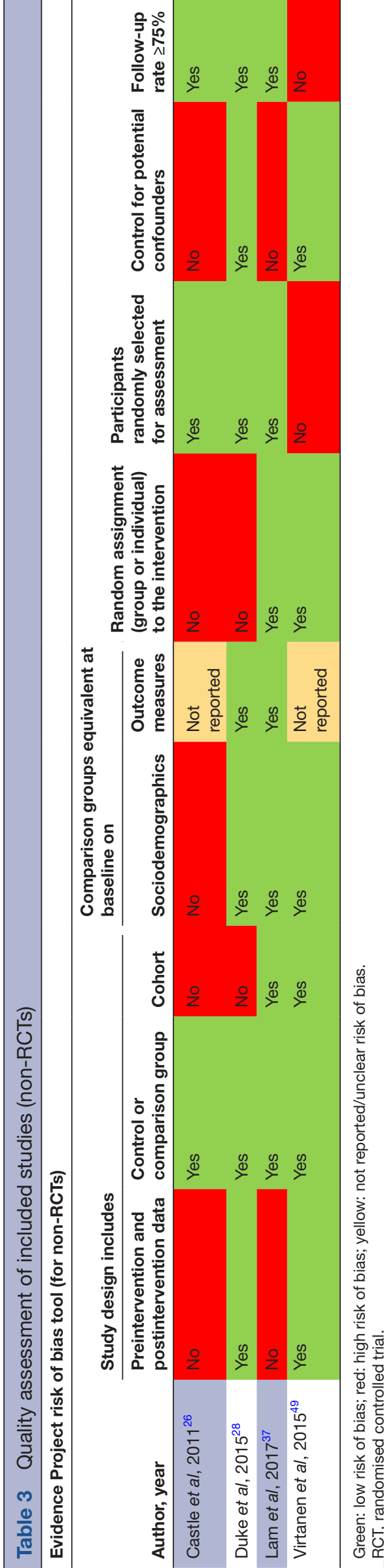

HPV self-test and cervical cancer education/promotion campaign, campaign alone and no intervention. ${ }^{28}$ In the community receiving self-test kits and the campaign, the cervical cancer screening rate increased by $15.2 \%$ $(p<0.001)$, compared with the campaign alone $(+2.9 \%$, $\mathrm{p}=0.07)$ and no intervention $(+8.5 \%, \mathrm{p}<0.001)$.

One RCT in Baltimore, Maryland, USA, examined the impact of HPV self-sampling among women living with HIV. $^{41}$ Women who self-collected cervicovaginal specimens for HPV testing in the HIV clinic were not more likely to complete cytology within 6 months compared with women who received only a reminder to make a cervical cancer screening appointment (22/63 vs 12/31, two-group test of proportions, $\mathrm{p}=0.59$, RR: $0.93,95 \% \mathrm{CI}$ 0.51 to 1.69$)$.

\section{Uptake stratified by setting}

Of the 29 included RCTs, 26 took place in high-income countries $^{21-25} 27$ 29-36 41-47 50-54 and 3 in LMICs ${ }^{38-40}$ as classified by the World Bank. In meta-analysis, HPV self-sampling was associated with increased uptake in both economic classifications, with greater impact in high-income countries (RR: 2.24, 95\% CI 1.86 to 2.71, I-squared: 99.38) than in LMICs (RR: $1.54,95 \%$ CI 1.01 to 2.34, I-squared: 98.43). Similarly, meta-analysis found increased uptake among HPV self-samplers regardless of setting, although potentially stronger effects were seen in urban areas (RR: 2.07, 95\% CI 1.54 to 2.83, I-squared: 99.21) $22313239-46485051$ and mixed urban/ rural areas (RR: $1.96,95 \%$ CI 1.45 to 2.65 , I-squared: 99.28) 21 23-25 $27293033-364548$ than in rural settings (RR: $1.40,95 \%$ CI 1.14 to 1.73 , I-squared: 89.69$).{ }^{3845485354}$

\section{Uptake stratified by sociodemographics}

Twelve RCTs were conducted solely among (or included disaggregated data for) women under 50 years of age $^{23} 24293334363839424345-4750$ and 11 RCTs among women over 50. ${ }^{23} 242934363839424345-4750$ In meta-analysis, HPV self-sampling showed a slightly stronger impact on uptake among older women (RR: 2.25, 95\% CI 1.44 to 3.50, I-squared: 99.18) than among younger women (RR: $1.95,95 \%$ CI 1.61 to 2.36, I-squared: 99.04). Four RCTs presented data for low socioeconomic status women ${ }^{24} 383947$ and three for high socioeconomic status women. ${ }^{24} 3947$ Meta-analysis found increased uptake after HPV self-sampling compared with standard-ofcare cervical cancer screening, both among low socioeconomic status women (RR: $1.62,95 \%$ CI 1.15 to 2.28 , I-squared: 96.24 ) and high (RR: $1.40,95 \%$ CI 1.15 to 1.71, I-squared: 27.65).

\section{Uptake stratified by supervision}

Two of the 29 included RCTs had participants self-collect samples for HPV testing under supervision; one used community health worker support for Hispanic, black and Haitian women self-sampling in Miami-Dade, Florida, USA, ${ }^{25}$ while the other used nurse support for women self-sampling in a poverty reduction programme 


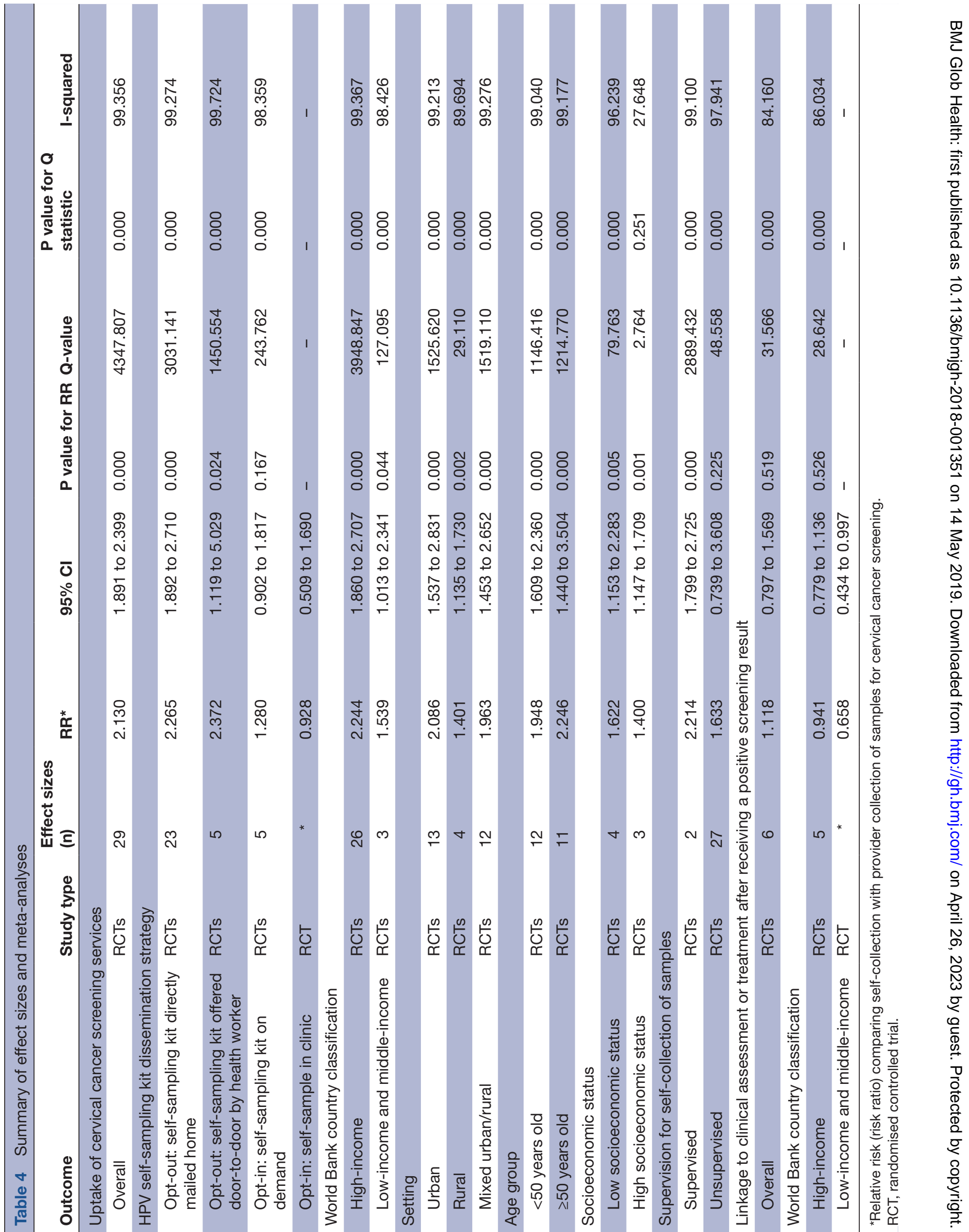




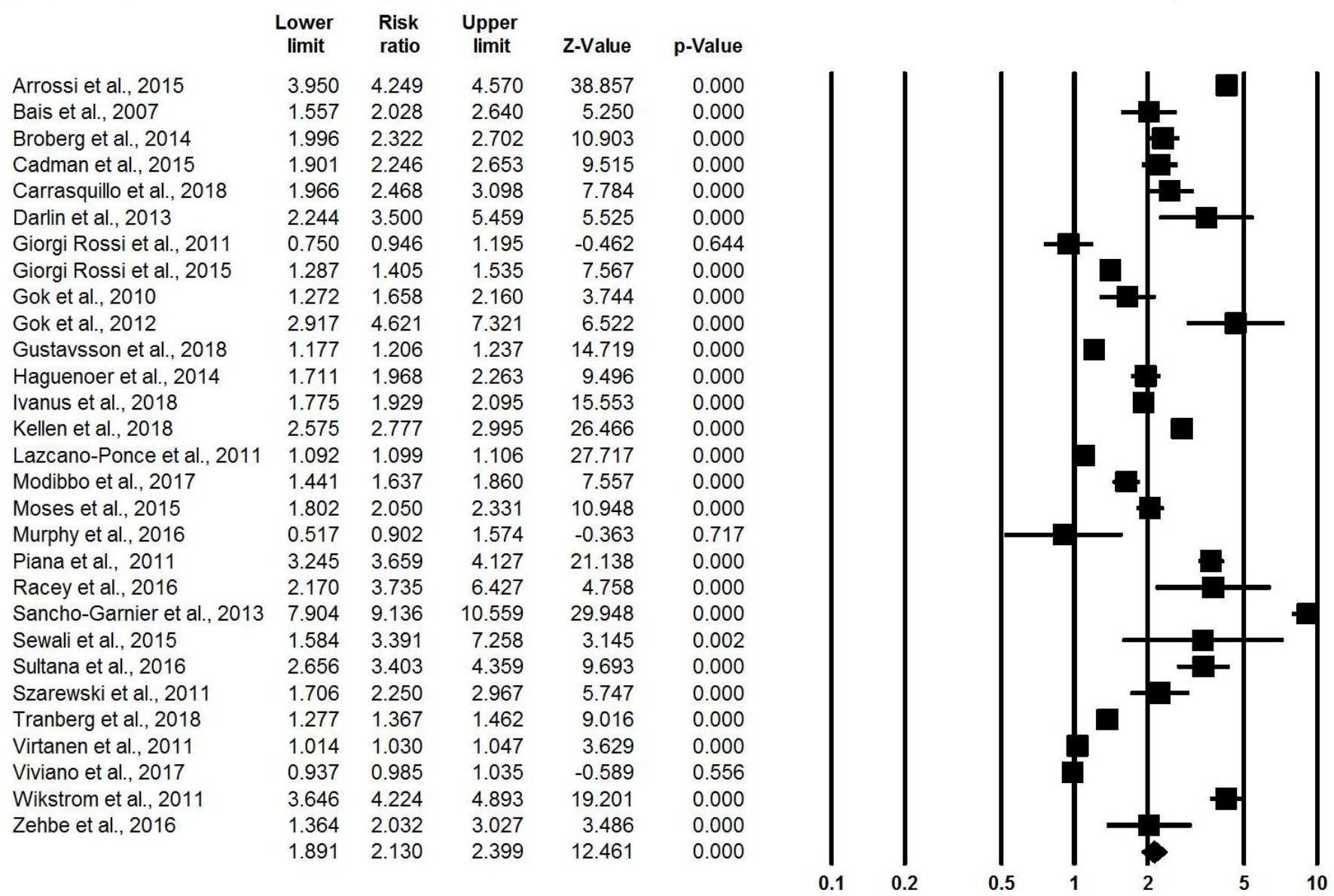

Figure 2 Meta-analysis showing relative risk of uptake of cervical cancer screening, overall.

in rural Mexico who had limited access to health services. ${ }^{38}$ Meta-analysis found greater uptake among supervised self-samplers (RR: $2.21,95 \%$ CI 1.80 to 2.73 , I-squared: 99.10$)^{2538}$ than among women self-sampling for HPV testing without supervision (RR: 1.63, 95\% CI 0.74 to 3.61, I-squared: 97.94$).^{21-242729-3639-4750-54}$

\section{Frequency of cervical cancer screening}

None of the included articles reported comparative data on frequency of cervical cancer screening.

\section{Social harms and adverse events}

None of the included articles compared outcomes relating to social harms and adverse events between HPV self-sampling and other cervical cancer screening services.

\section{Linkage to clinical assessment or treatment of cervical lesions}

Six of the included articles-five RCTs conducted among women who were not responding to standard screening programme algorithms in high-income settings in Europe who were mailed HPV self-sampling kits and one RCT offering HPV self-sampling door-to-door in Uganda-reported on linkage to clinical assessment or treatment of cervical lesions following a reactive self-test result and/or positive diagnosis for HPV by a healthcare provider. ${ }^{24} 2933404351$ Meta-analysis found no difference in the rate of postscreening linkage to care among women who received a positive screening result between arms (RR: $1.12,95 \%$ CI 0.80 to 1.57 , I-squared: 84.16 ) (figure 6 ). The study in a low-income country had potentially lower linkage to follow-up care (RR: $0.66,95 \%$ CI 0.43 to $1.00)^{40}$ compared with high-income countries (RR: 0.94, 95\% CI 0.78 to 1.14 , I-squared: 86.03) ${ }^{2429334351}$ although no appreciable difference was discerned in either setting nor either kit dissemination method.

\section{DISCUSSION}

Cervical cancer control is an essential priority for the WHO and many other partners. Available and accepted screening methods (ie, cytology, VIA or clinician-collected HPV testing) to detect women at risk for (pre-) cancer are not practical or accessible to the majority of women living in low-resource settings. Alternative screening strategies that decrease structural and other access challenges faced by women include self-sampling for HPV, as it is a simple intervention that women can use which supports their right to health. It also grants privacy and convenience to women, bypassing common barriers 
Study name

Bais et al., 2007

Broberg et al., 2014

Cadman et al., 2015

Darlin et al., 2013

Giorgi Rossi et al., 2011

Giorgi Rossi et al., 2015

Gok et al., 2010

Gok et al., 2012

Gustavsson et al., 2018

Haguenoer et al., 2014

Ivanus et al., 2018

Kellen et al., 2018

Modibbo et al., 2017

Piana et al., 2011

Racey et al., 2016

Sancho-Garnier et al., 2013

Sultana et al., 2016

Szarewski et al., 2011

Tranberg et al., 2018

Virtanen et al., 2011

Viviano et al., 2017

Wikstrom et al., 2011

Zehbe et al., 2016
Statistics for each study

$\begin{array}{crrrr}\begin{array}{c}\text { Lower } \\ \text { limit }\end{array} & \begin{array}{r}\text { Risk } \\ \text { ratio }\end{array} & \begin{array}{r}\text { Upper } \\ \text { limit }\end{array} & \text { Z-Value } & \text { p-Value } \\ 1.557 & 2.028 & 2.640 & 5.250 & 0.000 \\ 1.996 & 2.322 & 2.702 & 10.903 & 0.000 \\ 1.901 & 2.246 & 2.653 & 9.515 & 0.000 \\ 2.244 & 3.500 & 5.459 & 5.525 & 0.000 \\ 1.027 & 1.315 & 1.684 & 2.174 & 0.030 \\ 1.646 & 1.808 & 1.985 & 12.404 & 0.000 \\ 1.272 & 1.658 & 2.160 & 3.744 & 0.000 \\ 2.917 & 4.621 & 7.321 & 6.522 & 0.000 \\ 1.177 & 1.206 & 1.237 & 14.719 & 0.000 \\ 1.711 & 1.968 & 2.263 & 9.496 & 0.000 \\ 1.881 & 2.048 & 2.230 & 16.530 & 0.000 \\ 2.976 & 3.219 & 3.483 & 29.113 & 0.000 \\ 1.441 & 1.637 & 1.860 & 7.557 & 0.000 \\ 3.245 & 3.659 & 4.127 & 21.138 & 0.000 \\ 2.170 & 3.735 & 6.427 & 4.758 & 0.000 \\ 7.904 & 9.136 & 10.559 & 29.948 & 0.000 \\ 2.656 & 3.403 & 4.359 & 9.693 & 0.000 \\ 1.706 & 2.250 & 2.967 & 5.747 & 0.000 \\ 1.401 & 1.508 & 1.623 & 10.945 & 0.000 \\ 1.014 & 1.030 & 1.047 & 3.629 & 0.000 \\ 0.937 & 0.985 & 1.035 & -0.589 & 0.556 \\ 3.646 & 4.224 & 4.893 & 19.201 & 0.000 \\ 1.364 & 2.032 & 3.027 & 3.486 & 0.000 \\ 1.892 & 2.265 & 2.710 & 8.917 & 0.000\end{array}$

Risk ratio and $95 \% \mathrm{Cl}$

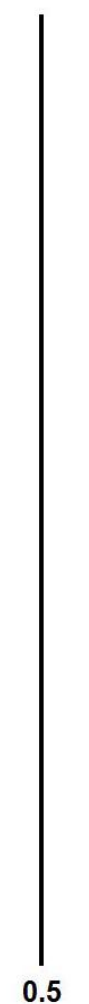

Figure 3 Meta-analysis showing relative risk of uptake of cervical cancer screening, opt-out (self-sampling kit directly mailed to home) versus control.

like fear, shame, geographical barriers, time limitations, cultural or religious considerations, and lack of access. ${ }^{55}$ Self-sampling has the potential to overcome the practical and personal barriers which may prevent some women from responding to standard cervical cancer screening.

Although interstudy heterogeneity was observed, the existing literature generally suggests that using HPV self-sampling methods, especially opt-out methods, increases uptake of cervical cancer screening services. When self-sampling kits were mailed directly to women's home addresses, meta-analysis of 22 RCTs showed that likelihood of attendance in the self-sampling arm was twice as high as the control. Data from observational studies corroborated these findings. Comparison of different strategies showed greater impact with HPV self-sampling offered door-to-door by health workers than by mailing kits directly to homes and by giving participants the responsibility to request or pick up kits, but the generally wide CIs around these estimates limit our ability to say for sure. Interestingly, in both opt-out strategies (mailed directly home and offered door-todoor), all effect sizes suggested that HPV self-sampling increased participation despite significant heterogeneity; when participants were given the choice to opt in to HPV self-sampling, the uptake outcome included mixed positive and negative effects. Quantitative heterogeneity in meta-analyses may be partially attributable to the use of RR for percentages instead of rates across
Study name

Arrossi et al., 2015
Carrasquillo et al., 2018
Lazcano-Ponce et al., 2011
Moses et al., 2015
Sewali et al., 2015

\begin{tabular}{cccrr}
\multicolumn{5}{c}{ Statistics for each study } \\
\cline { 2 - 4 } $\begin{array}{c}\text { Lower } \\
\text { limit }\end{array}$ & $\begin{array}{c}\text { Risk } \\
\text { ratio }\end{array}$ & $\begin{array}{c}\text { Upper } \\
\text { limit }\end{array}$ & Z-Value & p-Value \\
3.950 & 4.249 & 4.570 & 38.857 & 0.000 \\
1.966 & 2.468 & 3.098 & 7.784 & 0.000 \\
1.092 & 1.099 & 1.106 & 27.717 & 0.000 \\
1.802 & 2.050 & 2.331 & 10.948 & 0.000 \\
1.584 & 3.391 & 7.258 & 3.145 & 0.002 \\
1.119 & 2.372 & 5.029 & 2.253 & 0.024
\end{tabular}

Risk ratio and $95 \% \mathrm{Cl}$

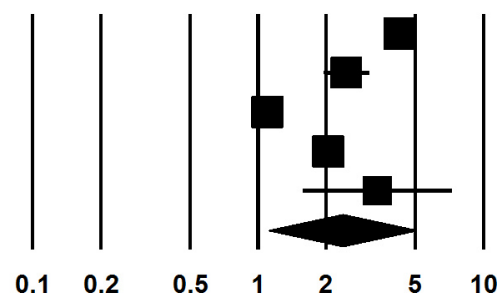

Figure 4 Meta-analysis showing relative risk of uptake of cervical cancer screening, opt-out (self-sampling kit offered door-todoor by health worker) versus control. 


\section{Lower Risk Upper}

limit ratio limit $Z-V a l u e ~ p-V a l u e$

$\begin{array}{lrrrr}\text { Giorgi Rossi et al., } 2011 & 0.4230 .581 & 0.798 & -3.354 & 0.001 \\ \text { Giorgi Rossi et al., } 2015 & 0.8991 .003 & 1.119 & 0.051 & 0.959 \\ \text { Ivanus et al., 2018 } & 1.7001 .849 & 2.012 & 14.325 & 0.000 \\ \text { Kellen et al., 2018 } & 2.1492 .333 & 2.534 & 20.110 & 0.000 \\ \text { Tranberg et al., 2018 } & 1.1331 .225 & 1.325 & 5.089 & 0.000 \\ & 0.9021 .280 & 1.817 & 1.382 & 0.167\end{array}$

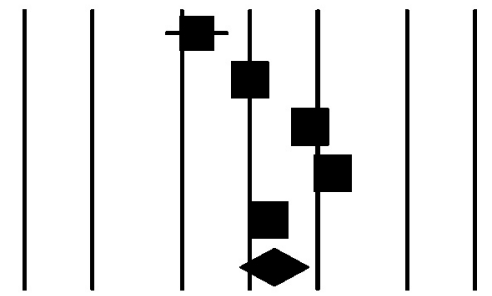

$\begin{array}{lllllll}0.1 & 0.2 & 0.5 & 1 & 2 & 5 & 10\end{array}$

Figure 5 Meta-analysis showing relative risk of uptake of cervical cancer screening, opt-in (self-sampling kit on demand) versus control.

studies, since high participation in control groups shifts the RR closer to 1 .

No studies offered comparative data for frequency of cervical cancer screening, nor for social harms and adverse events. Given that cervical cancer screening is recommended at relatively infrequent intervals, often up to 5 years, it is understandable that studies generally did not assess this longer term outcome. One follow-up study to an included RCT found that, on regular invitation to subsequent clinic-based cervical cancer screening 3 years after invitation to an HPV self-sampling trial, there was no statistically significant difference in participation rate (approximately 30\% in all arms) comparing those randomised to HPV self-sampling with HPV testing in clinic, whether prior self-sampling was conducted at home (RR: $1.05,95 \%$ CI 0.90 to 1.22 ) or at a pharmacy (RR: $0.96,95 \%$ CI 0.83 to 1.22$).{ }^{56}$ However, although the initial RCT compared self-sampling (sent kit home or offered free at local pharmacy) with standard population-based screening (sent reminder for cervical specimen taken at clinic), all women at the 3-year follow-up timepoint were reinvited for clinic-based HPV screening.

Finally, limited findings suggest that HPV self-sampling results in similar rates of linkage to clinical assessment or treatment of cervical lesions compared with standard services. No difference in linkage to follow-up diagnosis or treatment was found, regardless of kit dissemination method. Interestingly, compliance to follow-up testing and treatment was low in settings like France, ${ }^{43}$ where cervical screening programmes do not actively propose ascertainment appointments (eg, triage cytology, colposcopy) after positive initial screening results, but was relatively high in settings with organised programmes including active follow-up like England, ${ }^{24}$ Italy $^{29}$ and Sweden. ${ }^{33}$ While it is encouraging that linkage to care was not decreased among self-samplers, the generally low rate of clinical follow-up after a positive screening result is a cause for concern.

\section{Strengths and limitations}

This study had several strengths. We included both randomised and non-randomised studies, searched multiple databases, considered studies in any location or language, and used meta-analysis to present pooled effect sizes. Other reviews have examined the impact of HPV self-sampling on screening participation among women who are never or infrequently attending cervical cancer screening services, reporting that self-sampling has great potential to increase attendance among underscreened women compared with invitations to have a Pap test done by a health professional. ${ }^{12} 14-16$ This review expands on previous work by incorporating evidence from general populations and other segments of the population,

\section{Study name}

$\begin{array}{lrrrrr} & \begin{array}{c}\text { Lower } \\ \text { limit }\end{array} & \begin{array}{r}\text { Risk } \\ \text { ratio }\end{array} & \begin{array}{r}\text { Upper } \\ \text { limit }\end{array} & \text { Z-Value } & \text { p-Value } \\ \text { Cadman et al., 2015 } & 0.831 & 1.118 & 1.504 & 0.735 & 0.462 \\ \text { Giorgi Rossi et al., 2015 } & 3.245 & 6.823 & 14.347 & 5.064 & 0.000 \\ \text { Gustavsson et al., 2018 } & 1.004 & 1.068 & 1.136 & 2.071 & 0.038 \\ \text { Moses et al., 2015 } & 0.434 & 0.658 & 0.997 & -1.976 & 0.048 \\ \text { Sancho-Garnier et al., 2013 } & 0.405 & 0.738 & 1.346 & -0.992 & 0.321 \\ \text { Viviano et al., 2017 } & 0.589 & 0.880 & 1.314 & -0.625 & 0.532 \\ & 0.797 & 1.118 & 1.569 & 0.644 & 0.519\end{array}$

\section{Risk ratio and $95 \% \mathrm{Cl}$}

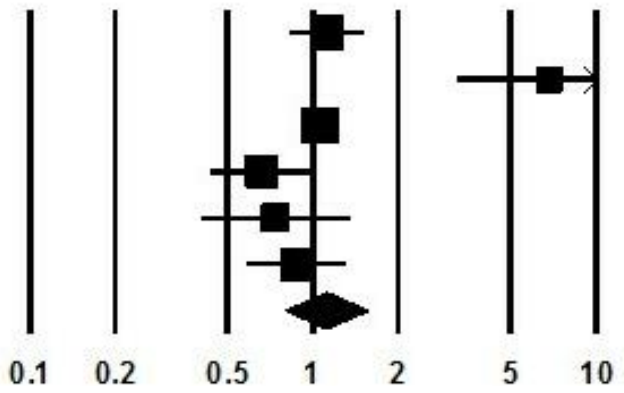

Figure 6 Meta-analysis showing relative risk of linkage to clinical assessment or treatment, self-sampling versus control. 
finding similar increases in uptake overall, not just among non-responders.

Our confidence in the findings is strengthened by the large number of rigorously conducted studies identified. However, despite identifying 34 articles which met our inclusion criteria, almost all of the included studies took place in high-income countries, where population-based cervical cancer screening programmes are more common. Additional research is needed to expand the evidence base in LMICs.

Further research should seek to answer questions surrounding how HPV self-sampling will be experienced by users in different settings and in different social and relationship contexts. For example, screening programmes may differ by the support available for self-sampling, mechanisms for test kit distribution and return, and linkages to diagnostic testing and subsequent triage to treatment. A systematic review of non-comparative or qualitative studies exploring users' experiences of self-sampling and impact on discrimination or other social harms may be valuable. Self-sampling for HPV testing can facilitate access to cervical cancer screening in low-resource settings, but more can be learnt about which characteristics of the self-sampling device or components of the test kit and supporting materials (eg, instructive images or video, inperson training or supervision) will increase acceptability among specific end-user groups like women living with HIV, from older age groups or with lower literacy. Work is needed to explore mechanisms and incentives for improved follow-up after screening, especially for populations self-sampling at home. The impact of large-scale use of HPV self-sampling for cervical cancer screening services on cost or resource use, and its cost-effectiveness, should likewise be examined. Operational research for scale-up of HPV self-sampling as part of self-care should be conducted, and clear guidelines for follow-ups for treatment should be developed.

The information presented in this review has been used to inform the development of WHO recommendations for self-care interventions for sexual and reproductive health and rights in relation to self-sampling for HPV testing. The benefits and harms of this intervention found in the present review can be considered along with values and preferences, resource use, human rights, and feasibility issues to inform the recommendation. It is hoped that additional research into the outcomes important to decision-makers will be conducted to fill the gaps identified in the current literature.

\section{CONCLUSION}

In summary, HPV self-sampling has the potential to increase uptake of cervical cancer screening. In meta-analysis, HPV self-sampling kits sent directly to women's homes or offered by a health worker resulted in greater uptake of screening, compared with standard of care, although subsequent attendance of cervical cancer services for follow-up clinical assessment or treatment was generally low and similar to other modes of cervical cancer screening. In addition, the size and direction of effect vary significantly among studies, so more research is needed to evaluate the feasibility, effectiveness and cost-effectiveness of self-sampling in specific local contexts prior to large-scale implementation of self-sampling, especially in LMICs where coverage of effective screening algorithms is currently low.

Acknowledgements We thank Laura Ferguson for her contribution to conceptualising this review, and Patricia Garcia, Gina Ogilvie and Nandi Siegfried for their thoughtful comments on the protocol. We thank Maribel Almonte, Nathalie Broutet and Anandi Yuvraj for reviewing an early draft of this manuscript. We also thank our Johns Hopkins Bloomberg School of Public Health graduate students (Melissa Camila Alamo, Chergai Gao Rittenberg, Priyanka Mysore, Holly Nishimura and Laura Graf) for their assistance in searching trial registries, screening citations and extracting data. Finally, we thank Janet Austin, Louise Cadman and Zahra Modibbo for graciously providing additional stratified data for our meta-analyses.

Contributors MN conceptualised the study. CEK and PTY designed the protocol, with feedback from HdV. PTY ran the search and oversaw screening, data extraction, and assessment of bias and quality of reporting. PTY and CEK conducted the meta-analysis. PTY drafted the manuscript. PTY, CEK, HdV and MN reviewed the draft, provided critical review, and read and approved the final manuscript. The corresponding author, as guarantor, accepts full responsibility for the finished article, has access to any data and controlled the decision to publish. The corresponding author attests that all listed authors meet the authorship criteria and that no others meeting the criteria have been omitted.

Funding We gratefully acknowledge financial support of the UNDP-UNFPAUnicef-WHO-World Bank Special Programme of Research, Development and Research Training in Human Reproduction (HRP) and the Children's Investment Fund Foundation (CIFF). HRP was involved in the study design. The funders played no part in the decision to submit the article for publication, nor in the collection, analysis and interpretation of data. All authors had full access to all of the data (including statistical reports and tables) in the study and can take responsibility for the integrity of the data and the accuracy of the data analysis.

Disclaimer This article is part of a series proposed UNDP/UNFPA/Unicef/WHO/ World Bank Special Programme for Human Reproduction (HRP) and commissioned by The BMJ. The BMJ retained full editorial control over external peer review, editing, and publication of these articles. Open access fees are funded by HRP.

Competing interests None declared.

Patient consent for publication Not required.

Provenance and peer review Not commissioned; externally peer reviewed.

Data availability statement Extracted data are available on request to the corresponding author

Open access This is an Open Access article distributed under the terms of the Creative Commons Attribution IGO License (https://creativecommons.org/ licenses/by-nc/3.0/igo/), which permits use, distribution, and reproduction for non-commercial purposes in any medium, provided the original work is properly cited.

\section{REFERENCES}

1. Chabra S. Cervical cancer preventable, treatable, but continues to kill women. Cervical Cancer 2016;1.

2. LaVigne AW, Triedman SA, Randall TC, et al. Cervical cancer in low and middle income countries: addressing barriers to radiotherapy delivery. Gynecol Oncol Rep 2017;22:16-20.

3. Bosch FX, Broker TR, Forman D, et al. Comprehensive control of human papillomavirus infections and related diseases. Vaccine 2013;31:I1-I31.

4. Elfström KM, Arnheim-Dahlström L, von Karsa L, et al. Cervical cancer screening in Europe: quality assurance and organisation of programmes. Eur J Cancer 2015;51:950-68.

5. Vaccarella S, Lortet-Tieulent J, Plummer M, et al. Worldwide trends in cervical cancer incidence: impact of screening against changes in disease risk factors. Eur J Cancer 2013;49:3262-73.

6. Spence AR, Goggin P, Franco EL. Process of care failures in invasive cervical cancer: systematic review and meta-analysis. Prev Med 2007;45:93-106. 
7. World Health Organization. Assessing national capacity for the prevention and control of noncommunicable diseases: report of the 2010 global survey. Geneva, Switzerland WHO; 2012. www.who.int/ cancer/publications/national_capacity_prevention_ncds.pdf

8. Liu G, Sharma M, Tan N, et al. HIV-positive women have higher risk of human papilloma virus infection, precancerous lesions, and cervical cancer. AIDS 2018;32:795-808.

9. Arbyn M, Ronco G, Anttila A, et al. Evidence regarding human papillomavirus testing in secondary prevention of cervical cancer. Vaccine 2012;30 Suppl 5:F88-F99.

10. Saslow D, Solomon D, Lawson HW, et al. American cancer Society, American Society for colposcopy and cervical pathology, and American Society for clinical pathology screening guidelines for the prevention and early detection of cervical cancer. CA Cancer J Clin 2012;62:147-72.

11. World Health Organization. Comprehensive cervical cancer control: a guide to essential practice (C4GEP) -. Second edition. Geneva, Switzerland: WHO, 2014

12. Arbyn M, Smith SB, Temin S, et al. Detecting cervical precancer and reaching underscreened women by using HPV testing on self samples: updated meta-analyses. BMJ 2018;8.

13. Harding-Esch EM, Hollis E, Mohammed $\mathrm{H}$, et al. Self-sampling and self-testing for STIs and HIV: the case for consistent nomenclature. Sex Transm Infect 2017;93:445-8.

14. Racey CS, Withrow DR, Gesink D. Self-collected HPV testing improves participation in cervical cancer screening: a systematic review and meta-analysis. Can J Public Health 2013;104:e159-66.

15. Snijders PJF, Verhoef VMJ, Arbyn M, et al. High-risk HPV testing on self-sampled versus clinician-collected specimens: a review on the clinical accuracy and impact on population attendance in cervical cancer screening. Int J Cancer 2013;132:2223-36.

16. Verdoodt F, Jentschke M, Hillemanns $P$, et al. Reaching women who do not participate in the regular cervical cancer screening programme by offering self-sampling kits: a systematic review and meta-analysis of randomised trials. Eur J Cancer 2015;51:2375-85.

17. Moher D, Liberati A, Tetzlaff J, et al. Preferred reporting items for systematic reviews and meta-analyses: the PRISMA statement. BMJ 2009;339:b2535.

18. Higgins JPT, Green S. Chapter 8.5 The Cochrane Collaboration's tool for assessing risk of bias. In: Higgins JPT, Altman DG, Sterne JAC, et al, eds. Cochrane Handbook for systematic reviews of interventions, version 5-1-0. 2011. London, England: The Cochrane Collaboration, 2011

19. Kennedy CE, Fonner VA, Armstrong KA, et al. The Evidence Project risk of bias tool: assessing study rigor for both randomized and nonrandomized intervention studies. Syst Rev 2019;8.

20. Borenstein M, Hedges L, Higgins JPT, et al. Comprehensive metaanalysis version 3 Biostat, Englewood, NJ, 2013. Available: https:// www.meta-analysis.com

21. Arrossi $S$, Thouyaret $L$, Herrero R, et al. Effect of self-collection of HPV DNA offered by community health workers at home visits on uptake of screening for cervical cancer (the EMA study): a population-based cluster-randomised trial. Lancet Glob Health 2015;3:e85-94.

22. Bais AG, van Kemenade FJ, Berkhof $\mathrm{J}$, et al. Human papillomavirus testing on self-sampled cervicovaginal brushes: an effective alternative to protect nonresponders in cervical screening programs. Int. J. Cancer 2007;120:1505-10.

23. Broberg G, Gyrd-Hansen D, Miao Jonasson J, et al. Increasing participation in cervical cancer screening: offering a HPV SelfTest to long-term non-attendees as part of RACOMIP, a Swedish randomized controlled trial. Int J Cancer 2014:134:2223-30.

24. Cadman L, Wilkes S, Mansour D, et al. A randomized controlled trial in non-responders from Newcastle upon Tyne invited to return a selfsample for human papillomavirus testing versus repeat invitation for cervical screening. J Med Screen 2015;22:28-37.

25. Carrasquillo O, Seay J, Amofah A, et al. HPV self-sampling for cervical cancer screening among ethnic minority women in South Florida: a randomized trial. J Gen Intern Med 2018;33:1077-83.

26. Castle PE, Rausa A, Walls T, et al. Comparative community outreach to increase cervical cancer screening in the Mississippi delta. Prev Med 2011;52:452-5

27. Darlin L, Borgfeldt C, Forslund $\mathrm{O}$, et al. Comparison of use of vaginal HPV self-sampling and offering flexible appointments as strategies to reach long-term non-attending women in organized cervical screening. J Clin Virol 2013;58:155-60.

28. Duke P, Godwin M, Ratnam S, et al. Effect of vaginal self-sampling on cervical cancer screening rates: a community-based study in Newfoundland. BMC Women's Health 2015;15.

29. Giorgi Rossi P, Fortunato C, Barbarino P, et al. Self-sampling to increase participation in cervical cancer screening: an RCT comparing home mailing, distribution in pharmacies, and recall letter. Br J Cancer 2015;112:667-75.

30. , Marsili LM, Camilloni L, et al. The effect of self-sampled HPV testing on participation to cervical cancer screening in Italy: a randomised controlled trial (ISRCTN96071600). Br J Cancer 2011;104:248-54.

31. Gok M, Heideman DAM, van Kemenade FJ, et al. HPV testing on self collected cervicovaginal lavage specimens as screening method for women who do not attend cervical screening: cohort study. BMJ 2010;340:c1040.

32. Gök M, van Kemenade FJ, Heideman DAM, et al. Experience with high-risk human papillomavirus testing on vaginal brush-based selfsamples of non-attendees of the cervical screening program. Int. J. Cancer 2012;130:1128-35.

33. Gustavsson I, Aarnio R, Berggrund M, et al. Randomised study shows that repeated self-sampling and HPV test has more than twofold higher detection rate of women with $\mathrm{CIN} 2+$ histology than Pap smear cytology. Br J Cancer 2018;118:896-904.

34. Haguenoer K, Sengchanh S, Gaudy-Graffin C, et al. Vaginal self-sampling is a cost-effective way to increase participation in a cervical cancer screening programme: a randomised trial. $\mathrm{Br} \mathrm{J}$ Cancer 2014:111:2187-96.

35. Ivanus $U$, Jerman T, Fokter AR, et al. Randomised trial of HPV selfsampling among non-attenders in the Slovenian cervical screening programme ZORA: comparing three different screening approaches. Radiol Oncol 2018;52:399-412.

36. Kellen E, Benoy I, Vanden Broeck D, et al. A randomized, controlled trial of two strategies of offering the home-based HPV self-sampling test to non- participants in the Flemish cervical cancer screening program. Int J Cancer 2018;143:861-8.

37. JUH Lam, Rebolj M, Møller Ejegod D, et al. Human papillomavirus self-sampling for screening nonattenders: Opt-in pilot implementation with electronic communication platforms. Int $J$ Cancer 2017;140:2212-9.

38. Lazcano-Ponce E, Lorincz AT, Cruz-Valdez A, et al. Self-collection of vaginal specimens for human papillomavirus testing in cervical cancer prevention (MARCH): a community-based randomised controlled trial. Lancet 2011;378:1868-73.

39. Modibbo F, Iregbu KC, Okuma J, et al. Randomized trial evaluating self-sampling for HPV DNA based tests for cervical cancer screening in Nigeria. Infect Agents Cancer 2017;12.

40. Moses E, Pedersen HN, Mitchell SM, et al. Uptake of communitybased, self-collected HPV testing $v s$. visual inspection with acetic acid for cervical cancer screening in Kampala, Uganda: preliminary results of a randomised controlled trial. Trop Med Int Health 2015;20:1355-67.

41. Murphy $\mathrm{J}$, Mark $\mathrm{H}$, Anderson $\mathrm{J}$, et al. A randomized trial of human papillomavirus self-sampling as an intervention to promote cervical cancer screening among women with HIV. J Low Genit Tract Dis 2016;20:139-44.

42. Piana L, Leandri F-X, Le Retraite L, et al. L'auto-prélèvement vaginal domicile pour recherche de papilloma virus haut risque. Campagne expérimentale du département des Bouches-du-Rhône. Bull Cancer Radiother 2011:98:723-31.

43. Sancho-Garnier H, Tamalet C, Halfon P, et al. HPV self-sampling or the Pap-smear: a randomized study among cervical screening nonattenders from lower socioeconomic groups in France. Int $J$ Cancer 2013;133:2681-7.

44. Sewali B, Okuyemi KS, Askhir A, et al. Cervical cancer screening with clinic-based Pap test versus home HPV test among Somali immigrant women in Minnesota: a pilot randomized controlled trial. Cancer Med 2015;4:620-31.

45. Sultana F, English DR, Simpson JA, et al. Home-based HPV selfsampling improves participation by never-screened and underscreened women: results from a large randomized trial (iPap) in Australia. Int J Cancer 2016;139:281-90.

46. Szarewski A, Cadman L, Mesher D, et al. HPV self-sampling as an alternative strategy in non-attenders for cervical screening - a randomised controlled trial. Br J Cancer 2011;104:915-20.

47. Tranberg M, Bech $\mathrm{BH}$, Blaakær J, et al. Preventing cervical cancer using HPV self-sampling: direct mailing of test-kits increases screening participation more than timely opt-in procedures - a randomized controlled trial. BMC Cancer 2018;18.

48. Tranberg M, Bech BH, Blaakær J, et al. HPV self-sampling in cervical cancer screening: the effect of different invitation strategies in various socioeconomic groups - a randomized controlled trial. Clin Epidemiol 2018;10:1027-36.

49. Virtanen A, Anttila A, Luostarinen T, et al. Improving cervical cancer screening attendance in Finland. Int $J$ Cancer 2015;136:E677-E684. 
50. Virtanen A, Nieminen P, Luostarinen T, et al. Self-sample HPV tests as an intervention for Nonattendees of cervical cancer screening in Finland: a randomized trial. Cancer Epidemiol Biomarkers Prev 2011;20:1960-9.

51. Viviano M, Catarino R, Jeannot E, et al. Self-sampling to improve cervical cancer screening coverage in Switzerland: a randomised controlled trial. Br J Cancer 2017;116:1382-8.

52. Wikström I, Lindell M, Sanner K, et al. Self-sampling and HPV testing or ordinary Pap-smear in women not regularly attending screening: a randomised study. Br J Cancer 2011;105:337-9.

53. Zehbe I, Jackson R, Wood B, et al. Community-randomised controlled trial embedded in the Anishinaabek cervical cancer screening study: Human papillomavirus self-sampling versus papanicolaou cytology. BMJ Open 2016;6:e011754.

54. Racey CS, Gesink DC, Burchell AN, et al. Randomized intervention of self-collected sampling for human papillomavirus testing in Under-Screened rural women: uptake of screening and acceptability. $J$ Womens Health 2016;25:489-97.

55. Braz NSDF, Lorenzi NPC, Sorpreso ICE, et al. The acceptability of vaginal smear self-collection for screening for cervical cancer: a systematic review. Clinics 2017;72:183-7.

56. Del Mistro A, Frayle H, Ferro A, et al. Efficacy of self-sampling in promoting participation to cervical cancer screening also in subsequent round. Prev Med Rep 2017;5:166-8. 